\title{
Mycobacterium tuberculosis cords within lymphatic endothelial cells to evade host immunity
}

\author{
Thomas R. Lerner, ${ }^{1}$ Christophe J. Queval, ${ }^{1}$ Rachel P. Lai, ${ }^{2}$ Matthew R.G. Russell, ${ }^{3}$ Antony Fearns, ${ }^{1}$ \\ Daniel J. Greenwood, ${ }^{1}$ Lucy Collinson, ${ }^{3}$ Robert J. Wilkinson, ${ }^{2,4,5}$ and Maximiliano G. Gutierrez ${ }^{1}$ \\ ${ }^{1}$ Host-pathogen interactions in tuberculosis laboratory, ${ }^{2}$ Tuberculosis laboratory, and ${ }^{3}$ Electron Microscopy Scientific \\ Technology Platform, The Francis Crick Institute, London, United Kingdom. ${ }^{4}$ Wellcome Centre for Infectious Diseases \\ Research in Africa, Institute of Infectious Disease and Molecular Medicine, and Department of Medicine, University of Cape \\ Town, Cape Town, Republic of South Africa. ${ }^{5}$ Department of Medicine, Imperial College London, London, United Kingdom.
}

\begin{abstract}
The ability of Mycobacterium tuberculosis to form serpentine cords is intrinsically related to its virulence, but specifically how $M$. tuberculosis cording contributes to pathogenesis remains obscure. Here, we show that several M. tuberculosis clinical isolates form intracellular cords in primary human lymphatic endothelial cells (hLECs) in vitro and in the lymph nodes of patients with tuberculosis. We identified via RNA-Seq a transcriptional program that activated, in infectedhLECs, cell survival and cytosolic surveillance of pathogens pathways. Consistent with this, cytosolic access was required for intracellular M. tuberculosis cording. Mycobacteria lacking ESX-1 type VII secretion system or phthiocerol dimycocerosates expression, which failed to access the cytosol, were indeed unable to form cords within hLECs. Finally, we show that M. tuberculosis cording is a size-dependent mechanism used by the pathogen to avoid its recognition by cytosolic sensors and evade either resting or IFN- $\gamma$-induced hLEC immunity. These results explain the longstanding association between $M$. tuberculosis cording and virulence and how virulent mycobacteria use intracellular cording as strategy to successfully adapt and persist in the lymphatic tracts.
\end{abstract}

Authorship note: TRL and CJQ contributed equally to this work.

Conflict of interest: The authors have declared that no conflict of interest exists.

Copyright: (c) 2020, American Society for Clinical Investigation.

Submitted: February 3, 2020

Accepted: April 15, 2020

Published: May 5, 2020.

Reference information: JCI Insight. 2020;5(10):e136937.

https://doi.org/10.1172/jci.

insight.136937.

\section{Introduction}

Mycobacterium tuberculosis is one of the deadliest bacterial pathogens of humankind and still constitutes a global health challenge (1). A striking phenotype of $M$. tuberculosis growing in nutrient broth is the ability of this pathogen to form serpentine cords, a morphological observation originally described by Robert Koch (2). This cording phenotype is intimately associated with virulence and immune evasion (3). In the mid1940s, Middlebrook, Dubos, and Pierce (4) provided the first morphological descriptions of M. tuberculosis growth in liquid and solid media, describing a distinct ability of tubercle bacilli to form large and elongated structures. Cording is a complex phenotype involving many mycobacterial factors including lipids, such as the "cord factor" glycolipid trehalose dimycolate (5-7), and a series of chemical modifications, such as cyclopropanation of mycolic acids in the cell wall (3).

Similar cording has been reported in other pathogenic mycobacteria, primarily in liquid media or extracellularly in various cell and organism models of infection. In zebrafish, $M$. abscessus released from apoptotic macrophages grows extracellularly, forming cords (8). It is postulated that apoptosis of infected macrophages is a key event in the release of bacteria within the extracellular space and subsequent initiation of cord formation. There are, however, a few reports showing that cording can also occur intracellularly. In 1928, Maximow and coworkers (9) first reported intracellular cording in tissue culture. In 1957, Shepherd (10) studied this phenomenon in HeLa cells and found that only fully virulent M. tuberculosis strains formed cords. More recently, Ferrer and coworkers (11) showed that an attenuated mutant of $M$. tuberculosis formed cords in fibroblasts.

Overall, extracellular cording has been shown in mycobacteria to be antiphagocytic and a trigger of extracellular trap formation in macrophages $(8,12,13)$. Although proposed as a virulence mechanism, this does not explain why an intracellular pathogen such as $M$. tuberculosis would prefer to replicate in cords in the relatively nutrient-poor extracellular space to avoid phagocytosis. 
Bacterial xenophagy is the process that regulates the removal of cytosolic bacteria after damage to phagosomal membranes during selective macroautophagy (14). This pathway constitutes one of the first cell autonomous defense pathways against intracellular pathogens $(15,16)$. A fraction of the $M$. tuberculosis population damages phagosomes to gain access to the cytosol and is subsequently recognized by autophagic adaptors and the xenophagy machinery. This process targets $M$. tuberculosis into autophagosomes and thus the lysosomal degradation pathway (17). Although there is a large body of literature demonstrating autophagy as an antimycobacterial pathway (18), recent evidence shows that $M$. tuberculosis can eventually block the fusion of autophagosomes with lysosomes $(19,20)$, and in mice, M. tuberculosis can evade autophagic responses in vivo (21).

Pulmonary tuberculosis (TB) is the most common form of the disease but lymphatics and lymph nodes are almost always systematically involved (22). Recent reports proposed that lymphatics may contribute to disease systemic dissemination and persistence $(23,24)$. M. tuberculosis mostly infects macrophages, although there is compelling evidence that a minor proportion of $M$. tuberculosis is found infecting various nonmyeloid cells in the lungs and lymph nodes in vivo (25-28). The role that these $M$. tuberculosis subpopulations play in TB pathogenesis in different cell types (e.g., immune vs. nonimmune) is unclear. We have previously shown in extrapulmonary TB that a subpopulation of $M$. tuberculosis is found in human lymphatic endothelial cells (hLECs) in lymph node biopsies and these cells could represent a reservoir for $M$. tuberculosis in infected patients (19).

Here, we discovered that $M$. tuberculosis forms large intracellular cords, consisting of up to thousands of individual bacteria arranged end to end, in hLECs and in biopsies of patients with TB. This intracellular cording phenotype is a common feature of virulent $M$. tuberculosis clinical isolates from the 4 human adapted strain lineages (1 to 4 ), whereas attenuated strains, those lacking either a functional type VII secretion system or phthiocerol dimycocerosate (PDIM) production, failed to form intracellular cords. We performed RNA-Seq analysis in the host environment during $M$. tuberculosis challenge and identified a transcriptional signature, from $M$. tuberculosis-infected hLECs, consistent with membrane damage and bacterial escape from the phagosome into the cytosol. We used correlative light electron microscopy (CLEM) to determine that the intracellular cords are formed of chains of individual M. tuberculosis, which are present only in the host cell cytosol, suggesting that cytosolic access is a prerequisite for intracellular bacterial cording. M. tuberculosis mutants lacking ESX-1 or PDIMs, which cannot access the cytosol, are indeed incapable of cording unless cocultured with WT bacteria to "smuggle" them from a shared phagosome into the cytosol. Finally, we show that cords are devoid of endosomal, phagosomal, and autophagosomal cellular markers and are formed from bacteria that successfully evaded p62-dependent xenophagy. Our results argue that intracellular cording represents an immune evasion strategy selected by virulent mycobacteria to survive within hLECs. When growing, the bacteria-forming cords, too large to be recognized by cytosolic sensors, represent a size-dependent mechanism that $M$. tuberculosis uses to avoid recognition and clearance by host degradative pathways such as xenophagy.

\section{Results}

M. tuberculosis forms extensive intracellular cords in hLECs and lymph node biopsies. By monitoring GFPexpressing $M$. tuberculosis H37Rv (GFP-M. tuberculosis) replication in hLECs at different time points after infection, we observed a striking ability of $M$. tuberculosis to form distinctive intracellular cords over the time (Figure 1A). 3D confocal imaging of $M$. tuberculosis-infected hLECs for 72 hours confirmed that these cords were intracellular rather than on the cell surface (Figure 1B). To quantitatively and accurately measure intracellular $M$. tuberculosis cording, we used the maximum Feret diameter, representing the distance between the 2 furthest extremities of the cord (Supplemental Figure 1; supplemental material available online with this article; https://doi.org/10.1172/jci.insight.136937DS1). As expected from Figure 1A, $M$. tuberculosis intracellular cords rapidly expended over time, reaching sizable Feret diameters up to $150 \mu \mathrm{m}$ only 72 hours after infection (Figure 1C). Intracellular cording was also observed in a human type II alveolar epithelial cell line (A549), although it was less prominent in A549 cells than in hLECs, likely due to the A549 cells themselves being smaller than hLECs (Figure 1D). Intracellular cord formation was present in the lab-adapted strain H37Rv (lineage 4) and when hLECs were infected with any of the 3 clinical isolates, N0072, N0145, N0024, representing $M$. tuberculosis lineages 1, 2, and 3 , respectively (Figure 1E). Importantly, the cords were also present in the lymph nodes of patients with extrapulmonary TB (Figure 1F). In Ziehl-Neelsen-stained lymph nodes with TB granulomas, we indeed 


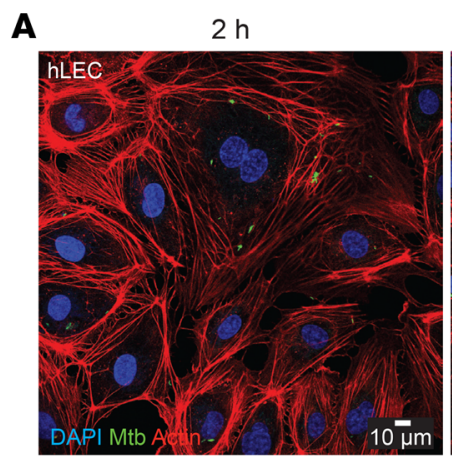

B

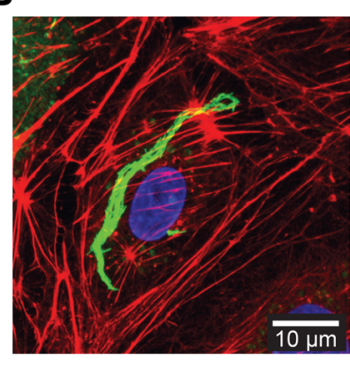

D

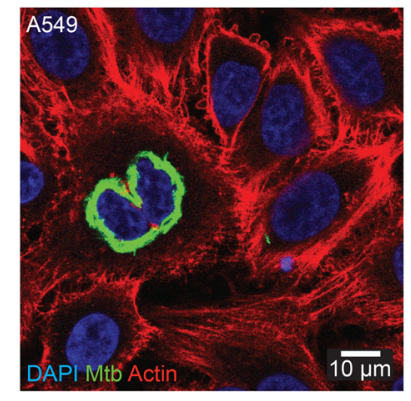

F
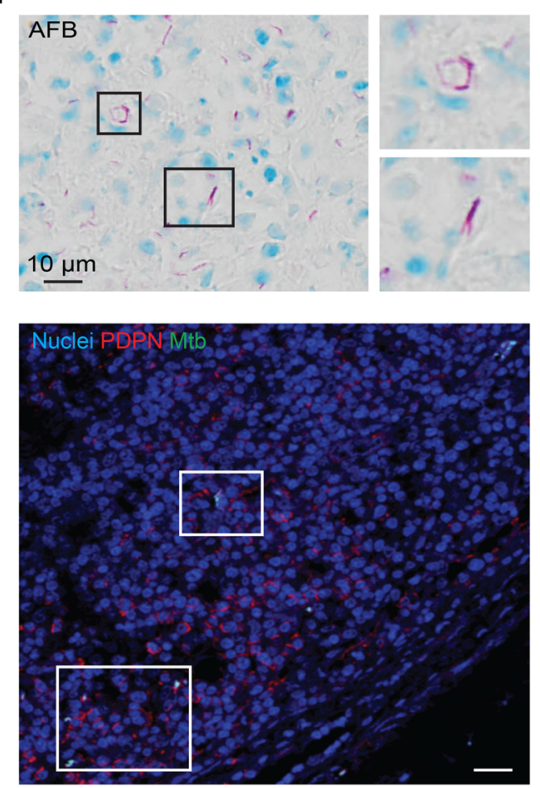

E
$24 \mathrm{~h}$

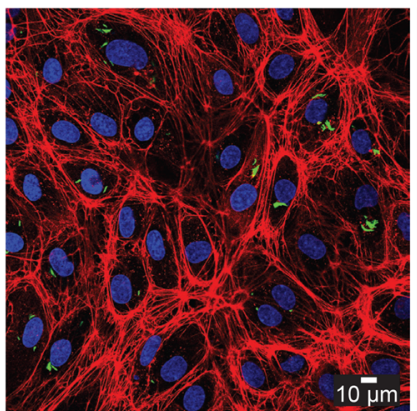

$10 \mu \mathrm{m}$
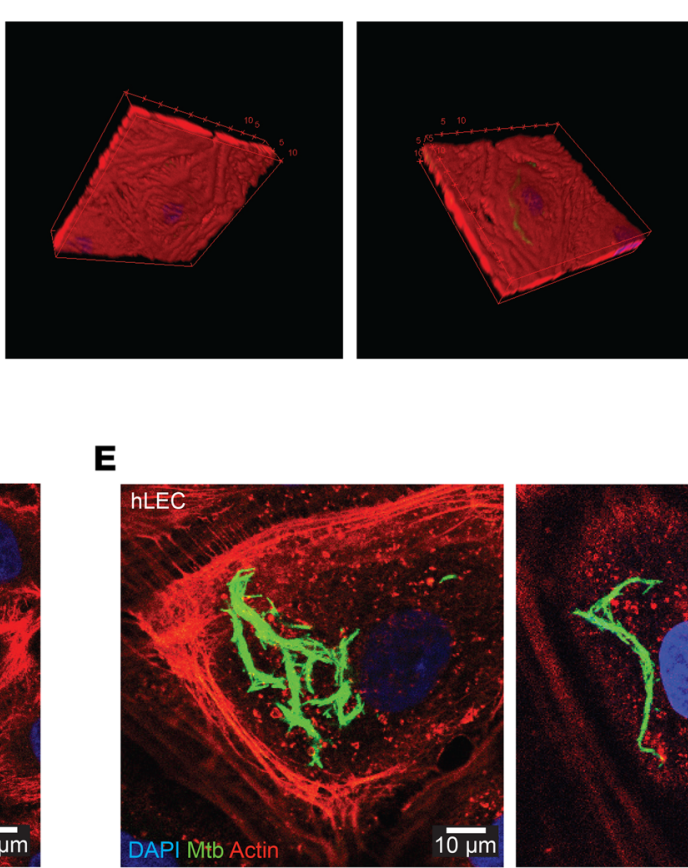

Lineage 1

N0072

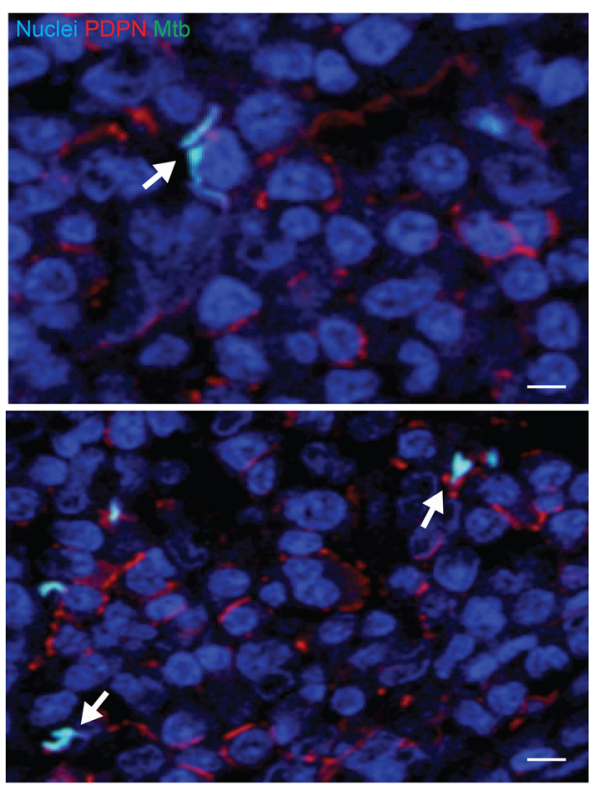

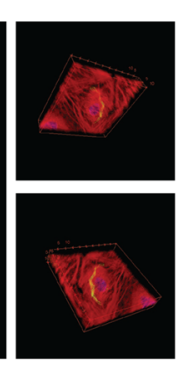

$72 \mathrm{~h}$
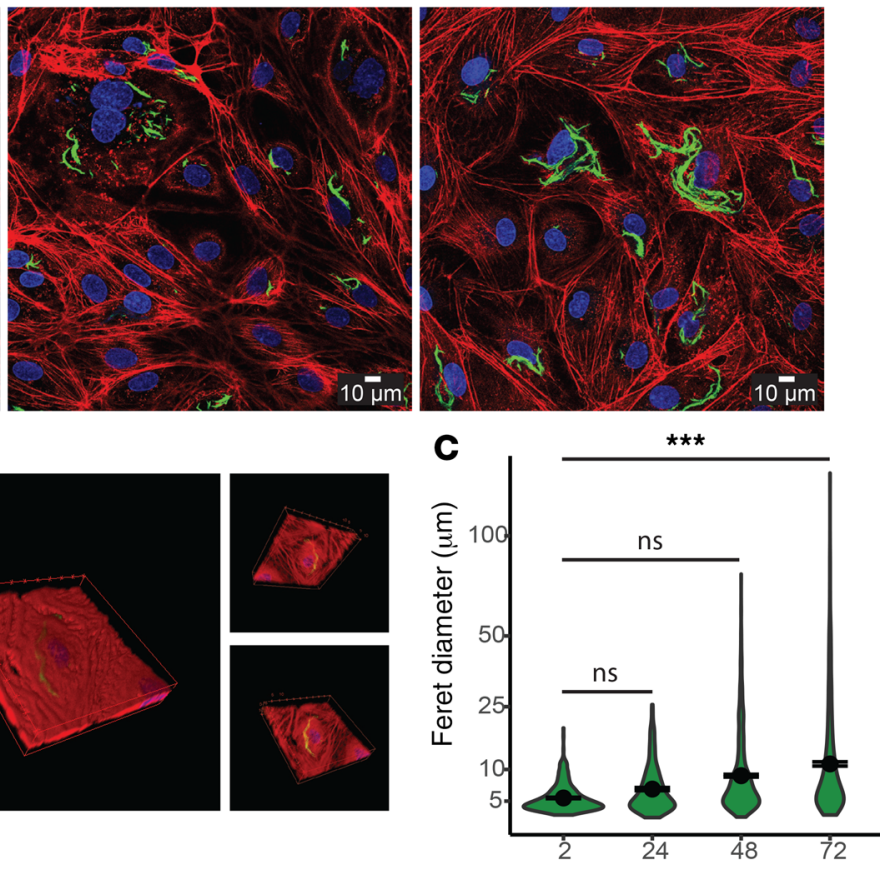

Hours after infection

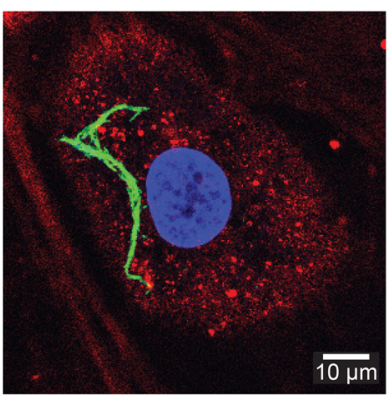

Lineage 2 N0145

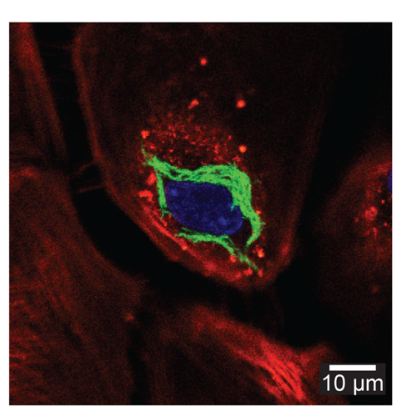

Lineage 3 N0024

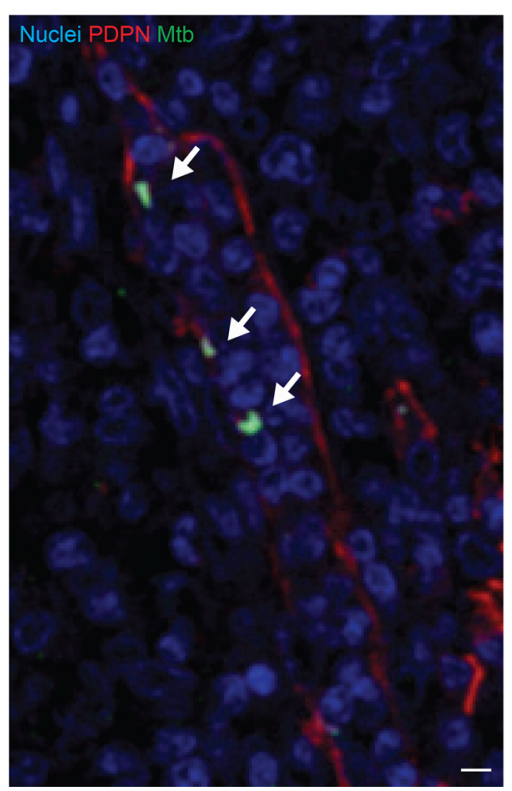


Figure 1. M. tuberculosis forms extensive intracellular cords in hLECs and lymph node biopsies. (A) Images of primary hLECs infected with GFP expressing M. tuberculosis for 2 to 72 hours. Over time, M. tuberculosis grows and forms large intracellular cords. Nuclei are stained with DAPI (blue) and F-actin is stained by rhodamine phalloidin (red). (B) 3D reconstruction of Z-stacks taken of an intracellular cord from (A). Various angles are shown to confirm that the cord is completely encapsulated within the host cell. (C) Measurement of the intracellular cords over time in hLECs (using the Feret diameter; see Supplemental Figure 1) showing that the cords elongated up to a maximum of $150 \mu \mathrm{m}$. The numbers of bacterial clusters analyzed were 418 ( 2 hours), 233 (24 hours), 814 (48 hours), and 618 (72 hours), obtained from 3 independent experiments. One-way ANOVA with Tukey's multiple comparisons tests: ${ }^{* *} P<0.001$. (D) Image of A549 cells infected with M. tuberculosis-EGFP for 72 hours showing an intracellular cord looping around the nucleus. Nuclei are stained with DAPI (blue) and F-actin is stained with rhodamine phalloidin (red). (E) Intracellular cord formation after 72 hours was also observed in hLECs infected with representative strains from 3 other M. tuberculosis lineages: N0072 (lineage 1), N0145 (lineage 2), and N0024 (lineage 3). Images displayed in $\mathbf{D}$ and $\mathbf{E}$ are representative of at least 3 independent experiments. (F) Tissue section of a granuloma present in a human lymph stained for AFB. Zoomed region shows association of M. tuberculosis cords with cells (black boxes). Representative histological sections from human patients after lymph node tissue resection surgery were stained for PDPN, M. tuberculosis, and nuclei (DAPI). Scale bar: $1 \mathrm{~mm}$. White boxes delimit the zoomed regions displayed on the right-hand side. Arrows indicate the presence of $M$. tuberculosis cords within PDPN+cells. Scale bar: $20 \mu \mathrm{m}$. hLECs, human lymphatic endothelial cells; AFB, acid fast bacilli; PDPN, podoplanin.

observed that intracellular bacterial cords were present in cells with pleiotropic morphologies, including endothelial-like morphology (Figure 1F). To confirm these observations, sections were stained for the lymphatic endothelial marker podoplanin (PDPN) and M. tuberculosis (19). Despite only a few LECs being infected with $M$. tuberculosis, the intracellular cording phenotype was associated with LECs in lymph node biopsies and the size of these cords ranged from 4 to $21 \mu \mathrm{m}$ (Figure 1F). Thus, the ability of M. tuberculosis to cord intracellularly in vitro in primary hLECs is not only conserved among the virulent human-adapted mycobacteria but also clinically relevant.

M. tuberculosis infection induces cytosolic surveillance of bacterial pathogens and prosurvival response in $h L E C S$. To better understand the host cell response to the extensive M. tuberculosis cording in the cytosol, we performed RNA-Seq analysis in uninfected and M. tuberculosis-infected hLECs 48 hours after infection, when cords started to become prominent. Among the top 10 statistically significant process networks induced by $M$. tuberculosis infection, we found groups of genes related to inflammation and interferon signaling, phagosome and antigen presentation, and innate immune response (Figure 2A). In addition to a strong proinflammatory response, we identified additional pathways that were significantly upregulated after infection, including cytosolic RNA and dsDNA sensing with an upregulation of type I IFN (Figure 2B). The pathways of cytosolic carbohydrate recognition as well as STING signaling were also upregulated, suggesting a high level of membrane damage induced by M. tuberculosis (Figure 2B). Importantly, RNA-Seq identified a transcriptional signature consistent with prosurvival pathways and antigen presentation (Figure 2C). This prosurvival signature was unexpected based on our data on human primary macrophages (29), although consistent with previous live-cell observations that active $M$. tuberculosis replication in primary hLECs was not associated with significant host cell death (19). We then confirmed by quantitative reverse-transcription PCR (RT-qPCR) the mRNA expression of the proinflammatory cytokine $I L 6$ as well as the type I IFN-responsive cytokines CXCL10 (IP10) and IFNB1 after infection in hLECs (Figure 2D). The prosurvival factors BCL2A1, EIF2AK2, and TNFAIP3 (A20); the cytosolic glycan-sensing genes galectin-3 (LGALS3), galectin-8 (LGALS8), and $c G A S$; and the foreign DNA sensor $Z B P 1$ were also significantly upregulated after infection (Figure $2 \mathrm{D}$ ). In the case of $L G A L S 3$, a high level of expression was already observed in uninfected cells (Figure 2D). Thus, infection of hLECs with $M$. tuberculosis induced host prosurvival pathways and negative regulators of cell death to protect the niche in which bacteria proliferate. On the other hand, endothelial cells upregulated cytosolic surveillance of RNA, DNA, and carbohydrates pathways to recognize M. tuberculosis in the cytosol.

M. tuberculosis intracellular cords are localized in the cytosol. Given the significant upregulation of cytosolic pathogen surveillance during cording in hLECs, we next sought to define the subcellular compartment within which $M$. tuberculosis cords. By using a correlative imaging approach, CLEM, we determined that $M$. tuberculosis intracellular cords were localized in the cytosol of hLECs in long structures that (in this example) looped around the host cell nucleus (Figure 3A). In contrast, small groups of $M$. tuberculosis containing relatively low numbers of individual bacteria were localized in a membrane-bound compartment (Figure 3B), as reported previously (19). The cords are usually formed of a bundle of several parallel chains of $M$. tuberculosis (Figure 3C), and therefore a single cord can consist of (up to) thousands of individual bacteria. We then measured, using 3D serial block face (SBF) CLEM, the volume of 25 individual bacteria (displayed as colored reconstructions) contained in a cord (Figure 3C) or in the membrane-bound small clump (Figure 3D). Interestingly, the volume of bacteria forming a cord was significantly lower than noncording bacteria (Figure 3E). This confirmed that the cords did not consist of 
A

\begin{tabular}{l|l|l}
\hline Network & $\%$ in Data & FDR \\
\hline Inflammation Interferon signaling & 50.0 & $1.890 \times 10^{-24}$ \\
Immune response Antigen presentation & 29.4 & $9.872 \times 10^{-13}$ \\
Immune response Innate immune response to RNA viral infection & 40.5 & $9.561 \times 10^{-12}$ \\
Immune response Th17-derived cytokines & 35.7 & $2.397 \times 10^{-10}$ \\
Inflammation IFN-gamma signaling & 32.1 & $5.936 \times 10^{-09}$ \\
Immune response Phagosome in antigen presentation & 23.5 & $1.434 \times 10^{-08}$ \\
Inflammation Jak-STAT Pathway & 25.3 & $3.250 \times 10^{-08}$ \\
Inflammation MIF signaling & 27.9 & $3.728 \times 10^{-08}$ \\
Inflammation IL-10 anti-inflammatory response & 33.3 & $4.600 \times 10^{-08}$ \\
Inflammation Innate inflammatory response & 25.0 & $7.400 \times 10^{-08}$
\end{tabular}

B

B
Proinflammatory
response
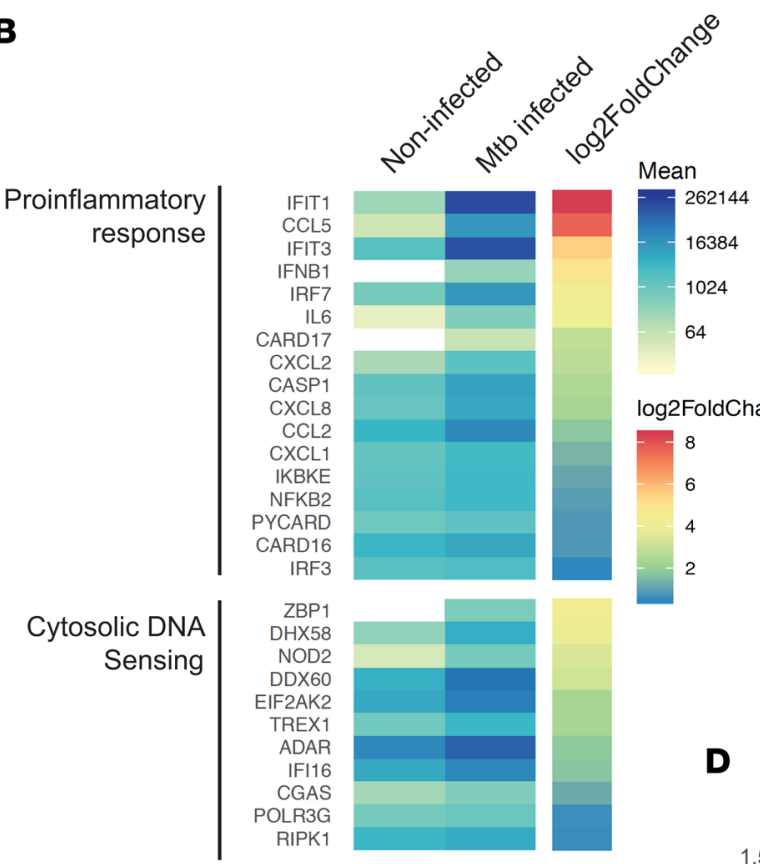

$$
\begin{array}{r}
\text { IFIT1 } \\
\text { CCL5 } \\
\text { IFIT3 } \\
\text { IFNB1 } \\
\text { IRF7 } \\
\text { IL6 } \\
\text { CARD17 } \\
\text { CXCL2 } \\
\text { CASP1 } \\
\text { CXCL8 } \\
\text { CCL2 } \\
\text { CXCL1 } \\
\text { IKBKE } \\
\text { NFKB2 } \\
\text { PYCARD } \\
\text { CARD16 }
\end{array}
$$

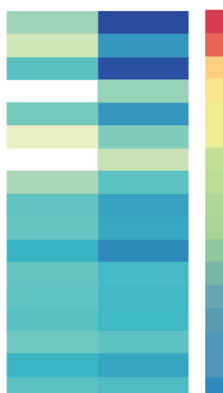

log2FoldChange

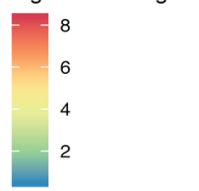

C

P

Pro-survival

Cytosolic RNA

Sensing
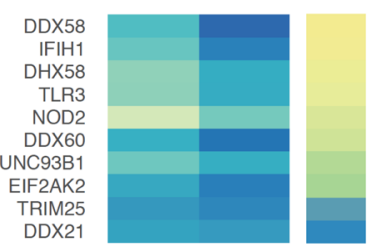

\begin{tabular}{r|r} 
Cytosolic Glycan & LGALS3BP \\
Sensing & LGALS17A \\
LGALS9 \\
LAMP3 \\
OPTN \\
LGALS8 \\
CALCOCO2
\end{tabular}

STING Signalling

INSIG1
TMEM173
TBK1
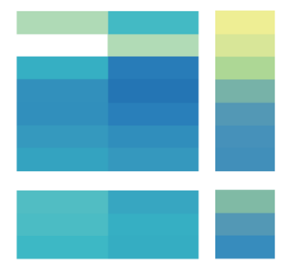

D
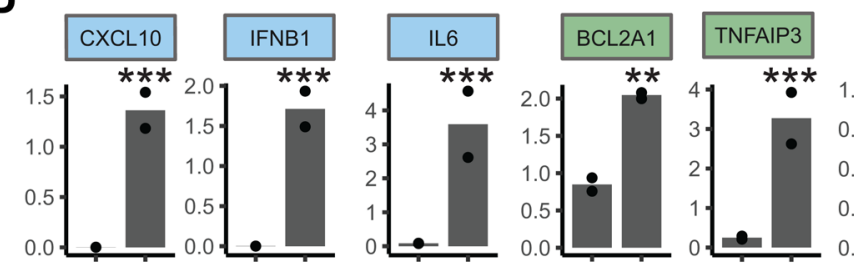

EIF2AK2
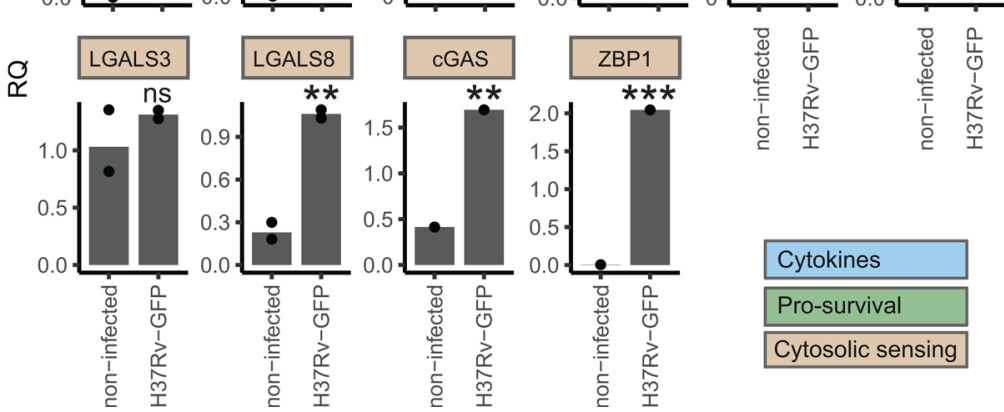

Mean

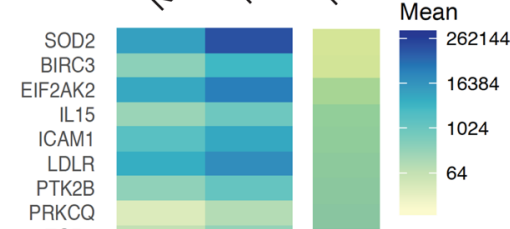

log2FoldChange

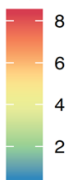

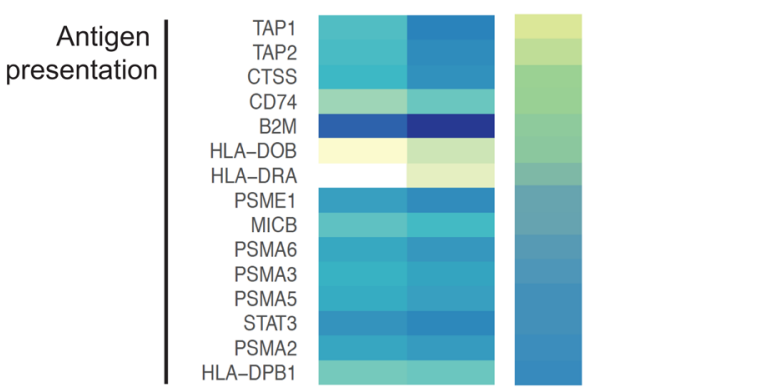

Figure 2. M. tuberculosis induces cytosolic surveillance and host prosurvival pathways. (A) Top 10 functional process analysis hits by FDR of genes significantly upregulated in hLECs 48 hours after infection, indicated by RNA-Seq. "\% in Data" indicates the \% of genes in the annotation group that were significantly upregulated in the analysis. (B) Heatmap of significantly upregulated (adjusted $P$ value $<0.05$ ) genes 48 hours after infection, grouped by sensing pathway, reveals an induction of proinflammatory, DNA, RNA, and glycan-sensing pathways and (C) genes involved in antigen presentation and the negative regulation of cell death. Significance of RNA-Seq data was calculated from 3 independent experiments. (D) qPCR confirmation of key infection response pathways 48 hours after infection. Data are representative of 3 independent experiments, each performed in triplicate. Student's $t$ tests: ${ }^{* *} P<0.01 ;{ }^{* *} P<0.001 ; \mathrm{ns}$, not significant. hLECs, human lymphatic endothelial cells. 
A

Fluorescence
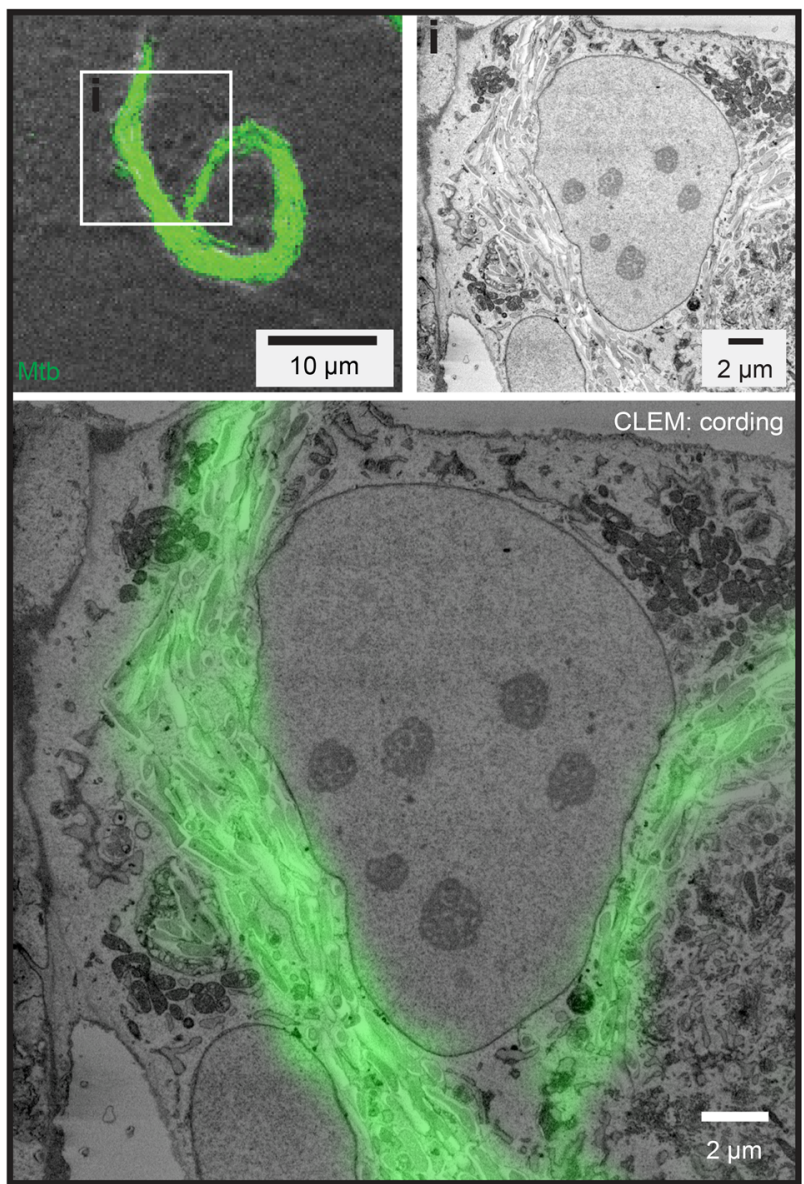

C SBF SEM: cording
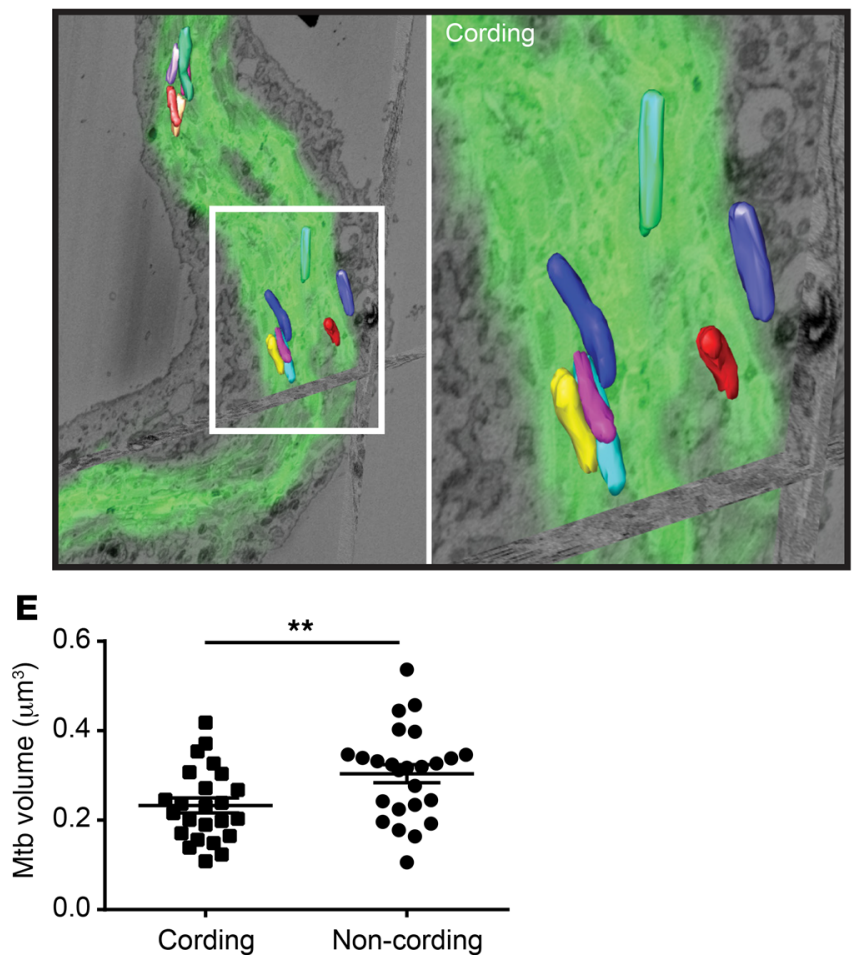

B

Fluorescence
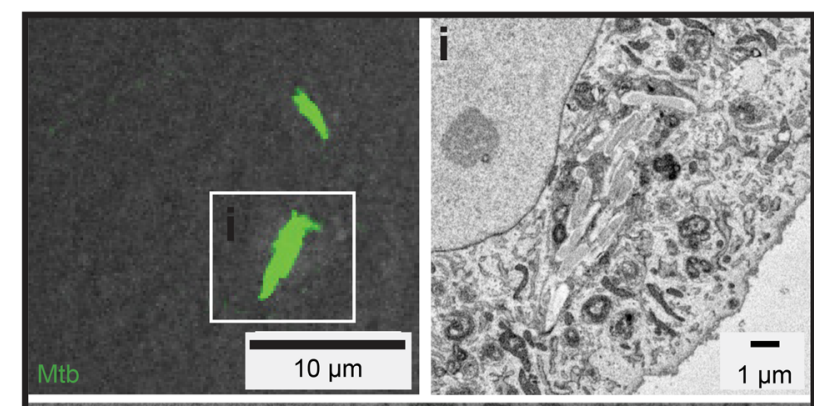

CLEM: non-cording

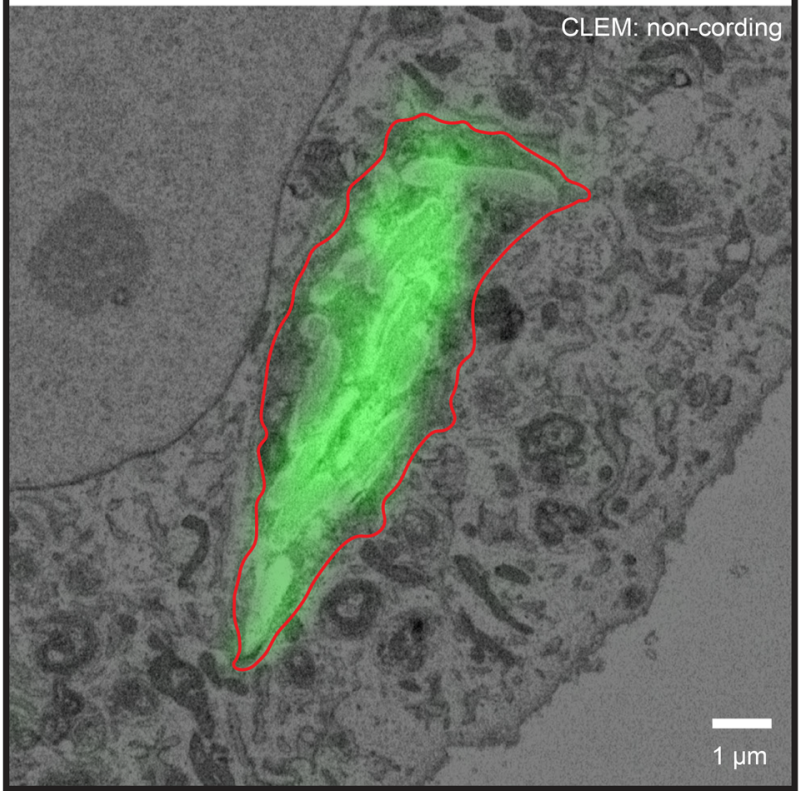

D SBF SEM: non-cording

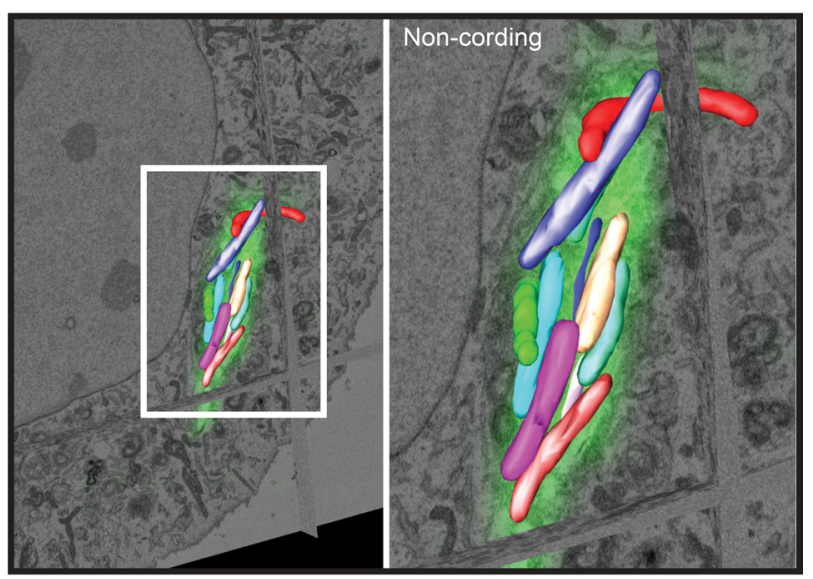


Figure 3. Intracellular cords are localized in the host cell cytosol and consist of chains of $\boldsymbol{M}$. tuberculosis of a small size. (A and B) CLEM images of hLECs infected with M. tuberculosis-GFP. Top left subpanel shows the light microscopy images, with the corresponding electron microscopy images in the top right subpanel. The larger subpanels show a composite of the fluorescence overlaid onto the electron microscopy. (A) M. tuberculosis intracellular cord, without any encapsulating host membrane, indicating that it is present in the cytosol. (B) M. tuberculosis encapsulated in a membranous compartment, as a control for confirming membrane preservation due to the sample preparation. Host cell membrane is highlighted in red. (C and D) To quantify the volume of $M$. tuberculosis, individual bacteria were manually segmented from slices of SBF SEM images and 3D reconstructions of selected bacteria were made (colored rods), using 3dmod. Representative reconstructions are shown, with corresponding fluorescence highlighted (matched manually with the corresponding SBF SEM slice in $Z$, and then aligned in xy with TurboReg in Fiji). Data set dimensions: (C) left panel, $8.7 \times 8.7 \times 50 \mathrm{~nm}$ pixels; right panel, $71.3 \times 71.36 \times 1 \mu \mathrm{m}$ in xyz; (D) left panel, $6.3 \times 6.3 \times 50 \mathrm{~nm}$ pixels; right panel, $51.6 \times 51.6 \times 2.75 \mu \mathrm{m}$ in $x y z$. (E) The volume of each bacterium reconstruction from 2 independent sample data sets was calculated in 3dmod, and a comparison between those in a membrane-bound compartment and those in an intracellular cord was made. The data \pm SEM show that individual bacteria forming cords are significantly smaller. Student's $t$ test: ${ }^{* *} P<0.01$. CLEM, correlative light electron microscopy; hLECs, images of human Iymphatic endothelial cells; SBF, serial block face; SEM, scanning electron microscopy.

abnormally long and filamentous mycobacteria but is more likely formed by actively replicating bacteria. We concluded that M. tuberculosis intracellular cording occurs in the cytosol of hLECs and that cytosolic M. tuberculosis cords are composed of hundreds or thousands of individual mycobacteria that are smaller than bacteria contained in membrane-bound compartments.

M. tuberculosis lacking RD1 locus or PDIM expression fails to cord within hLECs. We next sought to understand M. tuberculosis factors that contributed to the intracellular cording phenotype in hLECs. We have previously shown that the ESX-1 secretion system, encoded in the RD1 genomic region, and the cell wall lipid PDIMs are required for intracellular replication of $M$. tuberculosis in $\operatorname{hLECs}(19,30)$ as well as macrophages (31-33). Infection with the $M$. tuberculosis $\triangle \mathrm{RD} 1$ mutant that lacks the ESX-1 secretion system was not able to form cords but exhibited smaller clumps of bacteria instead, sometimes with a mesh-like appearance (Figure 4A). Similarly, the phenotype of the M. tuberculosis mutant lacking PDIM ( $\triangle$ PDIM) (31) also presented a clumpy, mesh-like phenotype, with an increased number of individual bacteria that were not organized in cords (Figure 4A). As shown Figure 1C, the Feret diameter of intracellular red fluorescent protein-expressing (RFP-expressing) M. tuberculosis (RFP-M. tuberculosis) WT increased over the time, whereas the size of both E2 Crimson- $\triangle \mathrm{RD} 1$ and GFP- $\triangle$ PDIM mutants remained significantly lower than WT strain, even after 3 days of infection (Figure 4B). Importantly, the lack of cording observed with the $\triangle R D 1$ mutant was not due to the reduced bacterial burden, since increasing the multiplicity of infection did not increase cord formation, although significant bacterial growth was observed (Figure 4, C-F). Moreover, we found that the upregulation of some genes in hLECs after infection (Figure 2B) such as IFNB1 or IL6 was RD1 and PDIM dependent (Supplemental Figure 2). For other genes, ESX-1 and PDIM seem to play a suppressive role, suggesting that other M. tuberculosis factors are involved in the activation of immune pathways. Altogether, these results confirm that intracellular cording in hLECs is specific to virulent $M$. tuberculosis strains. Attenuated M. tuberculosis strains, those lacking functional ESX1 secretion system or PDIMs, are indeed unable to cord within cells. Interestingly, it is known that both deletion of RD1 locus and inhibition of PDIM expression affect the ability of mycobacteria to escape from phagosome to reach the cytosol. Together with the exclusive cytosolic localization of virulent $M$. tuberculosis cords (shown Figure 3), the inability of those attenuated mutants to cord suggests that bacterial access to the cytosol may be required for the cording.

Access to the cytosol is required for $M$. tuberculosis replication and intracellular cording. The localization of M. tuberculosis cords suggested that the cytosol represents a permissive environment for M. tuberculosis replication; thus, we tested if the $\Delta \mathrm{RD} 1$ mutant of $M$. tuberculosis that is mostly localized in membrane-bound compartments could replicate and form intracellular cords if forced to access the cytosol. To achieve that, we performed a series of coinfection experiments combined with CLEM. As shown before, in RFP-M. tuberculosis H37Rv WT, single infection of hLECs with RFP-M. tuberculosis WT formed prominent intracellular cords, whereas single infection of hLECs with E2-Crimson-expressing M. tuberculosis $\triangle \mathrm{RD} 1$ or GFP-M. tuberculosis $\triangle \mathrm{PDIM}$ did not show cording (Figure 5A). Strikingly, if hLECs are coinfected with RFP-M. tuberculosis WT and E2-Crimson-expressing M. tuberculosis $\triangle \mathrm{RD} 1$ or GFP-M. tuberculosis $\triangle$ PDIM, the M. tuberculosis mutants lacking either ESX1 or PDIM were now able to clearly form intracellular cords (Figure 5A). Consistent with these observations, coinfection with $M$. tuberculosis WT partially restored the ability of $\triangle \mathrm{RD} 1$ or $\triangle \mathrm{PDIM}$ mutants to cord (Figure 5B). Although lower than the WT, the Feret diameter of both E2-Crimson-expressing $M$. tuberculosis $\triangle \mathrm{RD} 1$ or GFP-M. tuberculosis $\triangle \mathrm{PDIM}$ in coinfected cells was significantly increased compared with the 
A

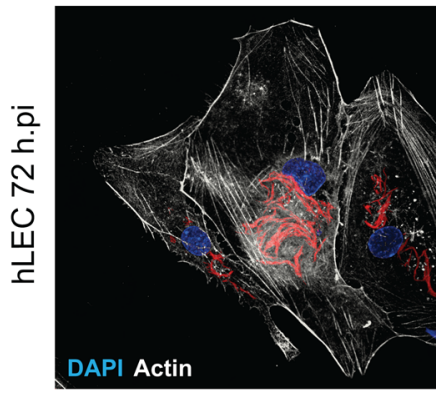

B

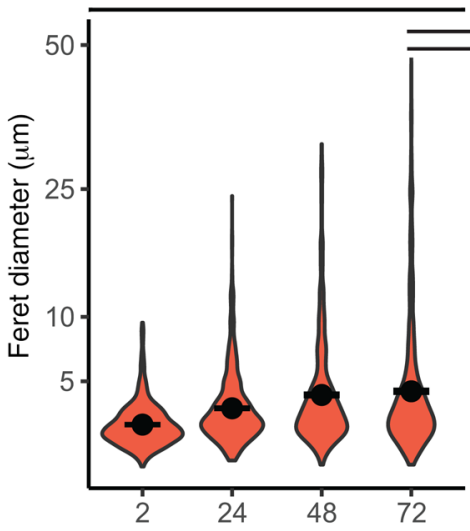

$\triangle P D I M$

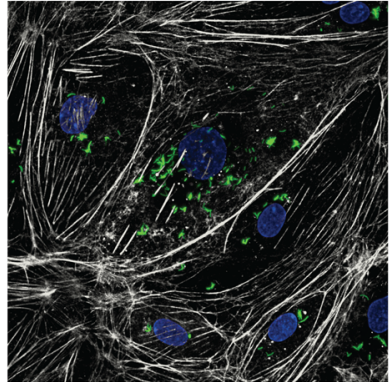

$\triangle \mathrm{PDIM}$

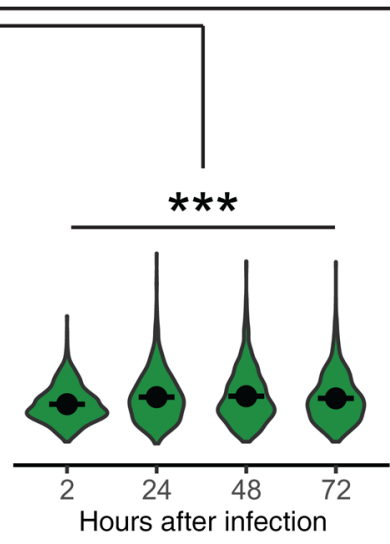

C

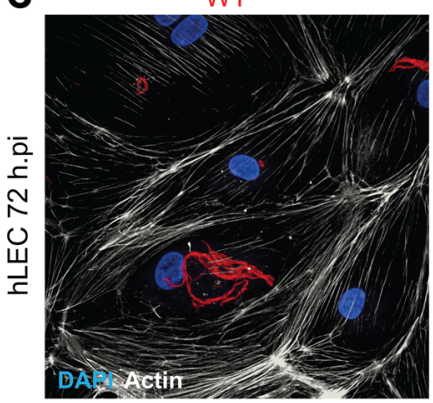

MOI:

10

D

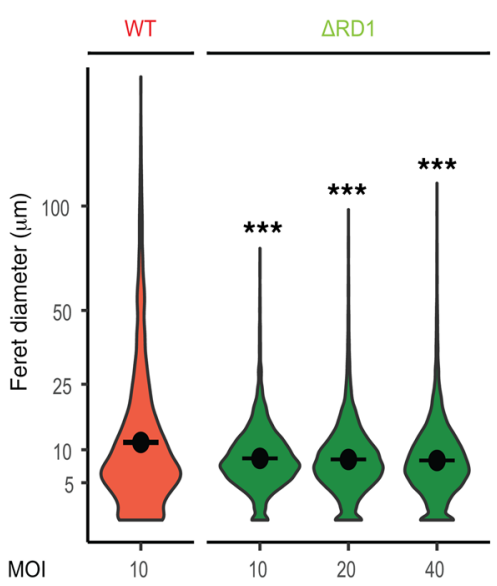

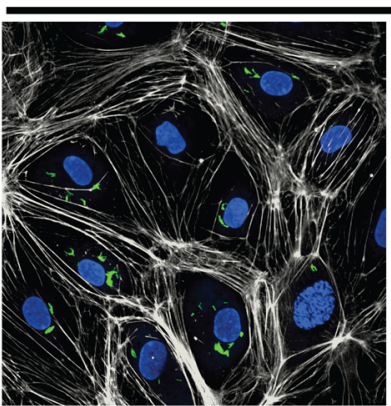

10

E

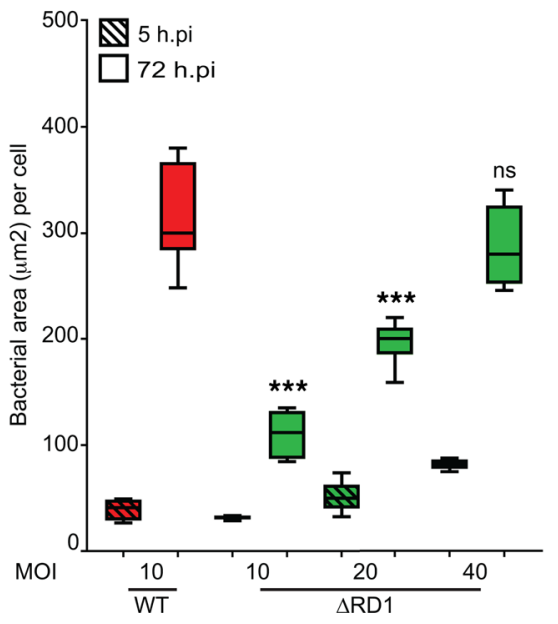

$\mathbf{F}$ $\triangle \mathrm{RD} 1$

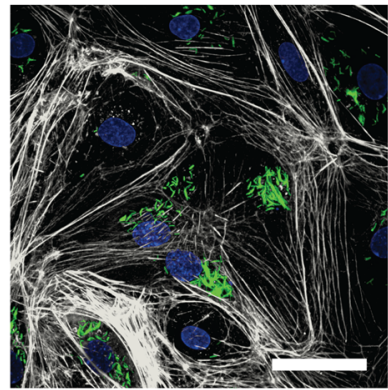

$\triangle \mathrm{RD} 1$

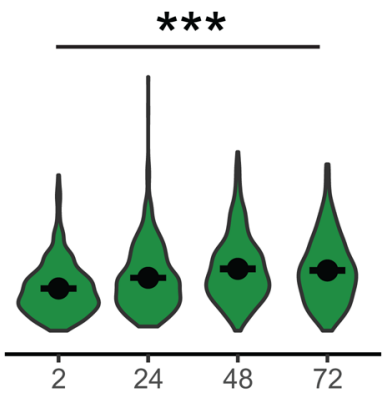

$\triangle \mathrm{RD} 1$

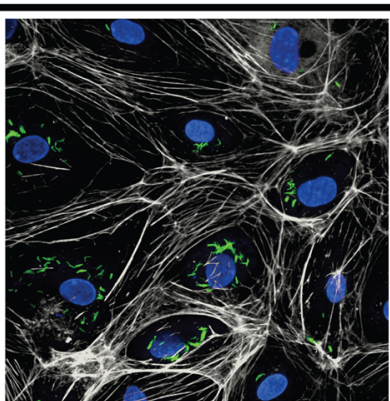

20

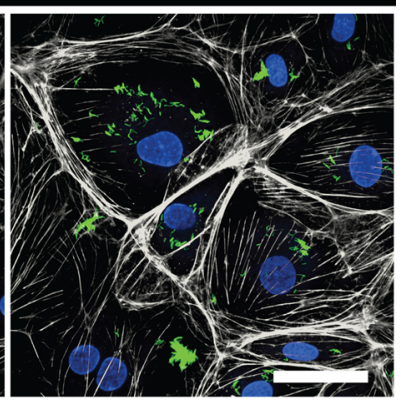

40

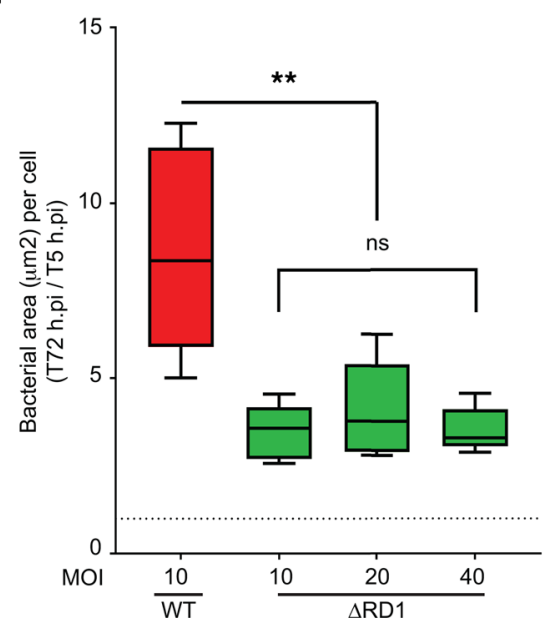

Figure 4. M. tuberculosis lacking RD1 locus or PDIMs expression fail to cord within hLECs. (A) hLECs were infected with RFP-expressing M. tuberculosis WT, GFP-expressing M. tuberculosis $\triangle P D I M$, or E2-Crimson-expressing $\triangle R D 1$ for 72 hours at a MOI of 10 , fixed and stained for F-actin with AF633 or AF488-phalloidin. Either deleting PDIM or the RD1 locus abolished cord formation. WT bacteria (red), $\triangle P D I M$ and $\triangle R D 1$-bacteria (green), F-actin (white), and nuclei (blue). Scale bar: $50 \mu \mathrm{m}$. (B) Feret diameter measurements from 3 independent experiments were plotted. For each condition tested, the number of 
bacterial clusters analyzed was between 600 and 1200. (C) hLECs were infected for 72 hours with RFP-expressing M. tuberculosis WT at a MOI of 10 or with E2-Crimson-expressing M. tuberculosis $\triangle \mathrm{RD1}$ at a MOI of 10, 20, or 40. WT bacteria (red), $\Delta \mathrm{RD1}$-bacteria (green), F-actin (white), and nuclei (blue). Scale bar: $50 \mu \mathrm{m}$. (D) Feret diameter measurements from images in C from 2 independent experiments were plotted. The numbers of bacterial clusters analyzed were 3,960 for WT and 6,470, 9,472, and 11,759 for $\triangle \mathrm{RD1}$ at MOI of 10, 20 and 40, respectively. (E) Quantification of the bacterial load per cell, expressed in bacterial area $\left(\mu \mathrm{m}^{2}\right)$ per cell, following the uptake ( 5 hours after infection) and 72 hours after infection. (F) Intracellular bacterial growth after 72 hours infection, expressed by the ratio bacterial area per cell 72 hours pi/5 hours pi. Values $>1$ represent the bacterial growth. (E and F) Data \pm SEM are representative of 2 independent experiments performed in duplicate. (B-E) One-way ANOVA with Tukey's multiple comparisons tests against $\mathrm{WT},{ }^{* *} P<0.01$; ${ }^{* * *} P<0.001 ; \mathrm{ns}$, not significant. PDIM, phthiocerol dimycocerosate; hLECs, images of human lymphatic endothelial cells; pi, postinfection.

single infection condition (Figure 5B). Importantly, in coinfected cells, both $M$. tuberculosis $\Delta \mathrm{RD} 1$ and $\triangle \mathrm{PDIM}$ were able to replicate more efficiently (Figure 5C). Using CLEM, we confirmed that RFP- $M$. tuberculosis WT was localized in the cytosol and defined at the ultrastructural level and that the cords formed by GFP-M. tuberculosis $\triangle \mathrm{RD} 1$ in coinfected cells were now localized in the cytosol (Figure 5, D and E). Altogether, our data demonstrate that forcing the access to the cytosol of attenuated $\Delta \mathrm{RD} 1$ or $\triangle$ PDIM mutants by coinfection with $M$. tuberculosis WT strain restored both their intracellular replication and their ability to cord intracellularly. This confirms that the effect of ESX-1 or PDIM expression on intracellular cording is mediated by access to the cytosol.

Intracellular cords are not recognized by cytosolic immune sensors in both resting and IFN- $\gamma$-activated hLECs. Bacterial access to the cytosol is essential for the replication of virulent mycobacteria, which, when exposed to the cytosolic components, must avoid being recapture by host-immune sensors and targeted for degradation. In hLECs, M. tuberculosis targeting via selective autophagy is PDIM dependent (30) and entirely RD1 dependent (Supplemental Figure 2), suggesting that, in hLECs, xenophagy primarily recognizes mycobacteria that access the cytosol. As cytosolic location is a prerequisite for mycobacterial cording in hLECs, we hypothesized that bacterial cords originate from bacteria, which successfully evade host-degradative pathways such as xenophagy. We thus examined the dynamics of intracellular bacterial growth and its association with the autophagic marker p62 (Figure 6, A-C; and Supplemental Videos 1-3). Live-cell imaging of $M$. tuberculosis-infected hLECs expressing RFP-p62 revealed that the intracellular cords form from bacteria, which either have completely evaded p62-positive compartments (Figure 6A and Supplemental Video 1) or have initially been growth-restricted in a p62-positive state (Figure 6B) but subsequently became p62-negative, in which this process can also cycle several times (Figure 6C and Supplemental Video 2). Crucially, the $M$. tuberculosis cords only ever form once the bacteria have lost p62 (Figure 6C and Supplemental Video 3), suggesting that cording is a consequence of avoiding an autophagic state or that cord formation blocks autophagic targeting, potentially by being too large to encapsulate and recapture from the cytosol. Thus, we investigated whether cording versus noncording populations of $M$. tuberculosis present in hLECs 72 hours after infection were differentially recognized by the selective autophagy machinery. When we colabeled ubiquitin and p62 in cord-containing cells, we notably found that both markers selectively associated with mostly small bacterial clusters but not with $M$. tuberculosis cords, as defined by having a Feret diameter greater than $10 \mu \mathrm{m}$ (Figure 6, D and E). Importantly, large M. tuberculosis cords did not associate with selective autophagy markers Galectin-8, NDP52, and LC3B as well as the late endosomal/lysosomal markers LAMP-2 and cathepsin D, whereas single or small groups/clumps of bacteria (Feret diameter $<10$ $\mu \mathrm{m}$ ) presented a higher association with all those markers (Figure 6E). These data indicated that cytosolic $M$. tuberculosis cords were not recognized by xenophagy or any other immune sensor tested.

We have previously reported that, in resting hLECs, autophagy is not necessarily detrimental for virulent mycobacteria, but when activated with IFN- $\gamma$ before infection, the autophagy pathway restricted bacterial growth and promoted bacterial clearance (19). We then examined whether intracellular cords could still evade cytosolic detection by autophagy machinery in IFN- $\gamma$-activated hLECs. As observed in resting hLECs, mostly small bacterial clusters were targeted by autophagic markers LC3B and p62, whereas intracellular cords were not recognized by any of them (Figure 6, F and G). These data confirm that intracellular cording is an effective mechanism used by virulent mycobacteria to evade hLECs' immune defenses and to persist within lymphatic endothelium.

\section{Discussion}

Since the identification of $M$. tuberculosis as the etiologic agent of human TB, the phenomenon of cording has attracted significant interest because of its association with virulence and infection in vivo. Whereas there are many studies that implicate extracellular cording as a mechanism to subvert phagocytosis, there is 
A

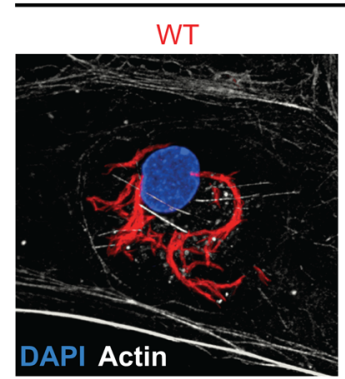

single

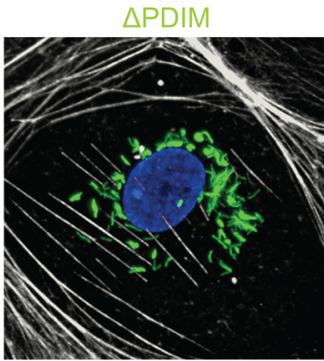

$\triangle \mathrm{RD} 1$

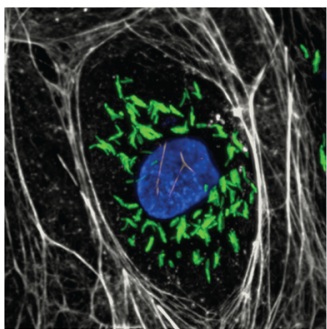

co-inf

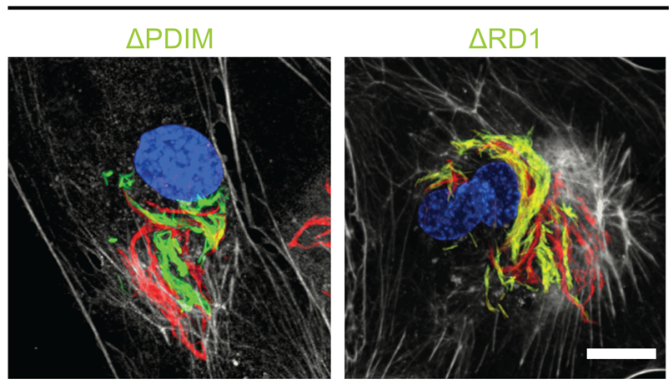

B $\mathrm{n}=1,152 \quad 10,1317,1272,3653,221$

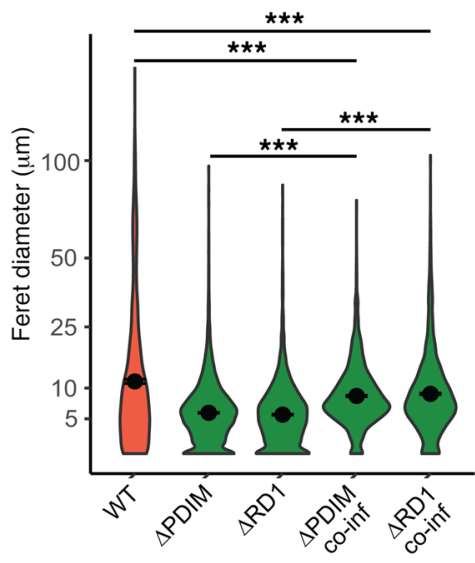

C

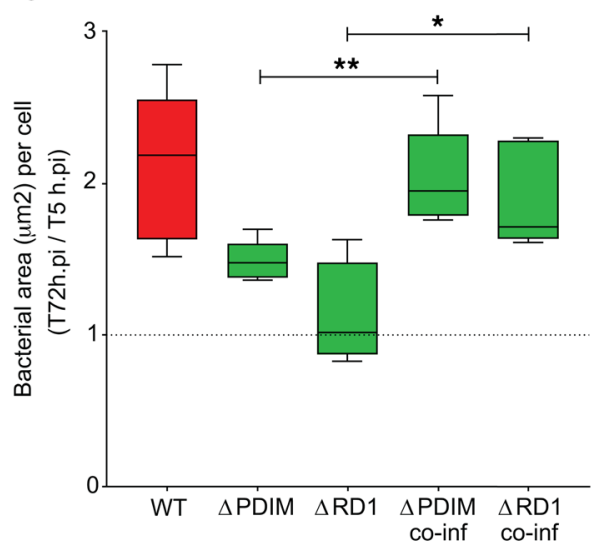

D

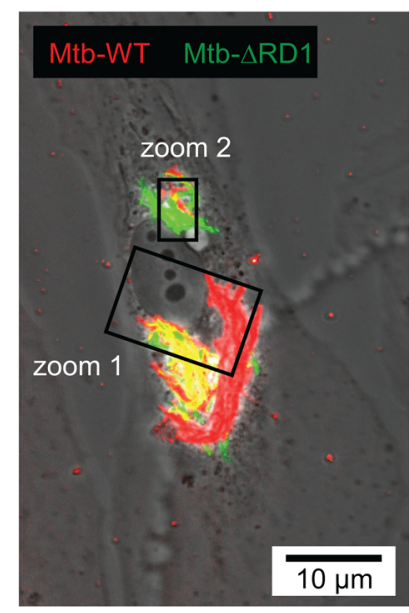

\section{CLEM}
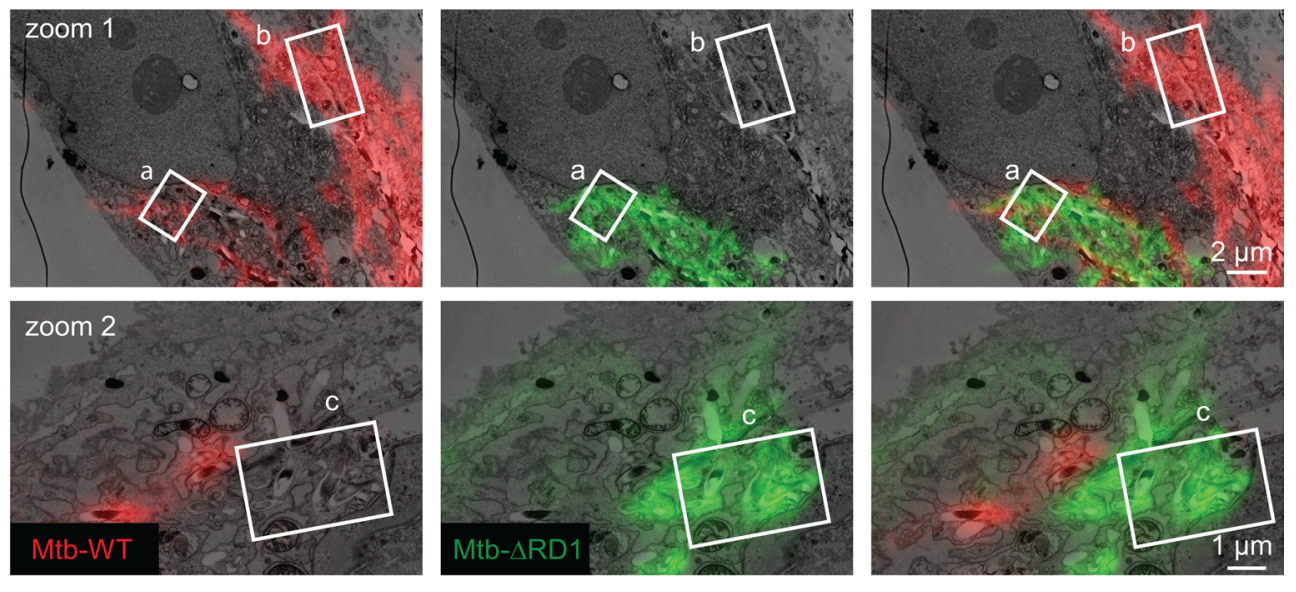

E TEM
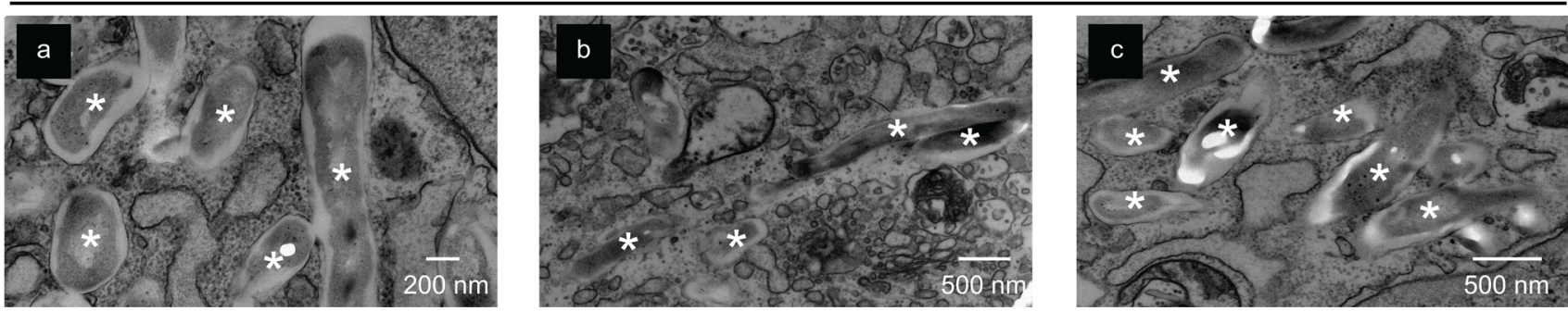

Figure 5. Access to the cytosol is required for M. tuberculosis intracellular cording. (A) hLECs were infected for 72 hours with $M$. tuberculosis WT-RFP (red), M. tuberculosis- $\triangle$ PDIM-GFP (green), and M. tuberculosis- $\triangle$ RD1-E2-Crimson (green) either individually or as a coinfection WT-RFP/ $\triangle \mathrm{PDIM-GFP} \mathrm{or}$ WT-RFP/ARD1-E2-Crimson. Cells were then fixed and stained for F-actin with AF633 or AF488-phalloidin (both visualized in white) and DAPI (blue). Scale bar: $10 \mu \mathrm{m}$. The images show that during single infection, M. tuberculosis WT exhibited intracellular cording, whereas $M$. tuberculosis $\triangle \mathrm{PDIM}$ or $\triangle$ RD1 did not. However, in the coinfected sample, both $M$. tuberculosis $\triangle \mathrm{PDIM}$ and $\triangle \mathrm{RD} 1$ were able to form intracellular cords. (B) Feret diameter measurements from images in $\mathbf{A}$ were plotted. $n$ represents the number of bacterial clusters analyzed. (C) Intracellular bacterial growth 72 hours after 
infection, expressed by the ratio bacterial area per cell 72 hours pi/5 hours pi. Values $>1$ represent the bacterial growth. (A-C) Data \pm SEM are representative of 3 independent experiments. One-way ANOVA with Tukey's multiple comparisons tests: ${ }^{*} P<0.05 ;{ }^{* *} P<0.01$; ${ }^{* * *} P<0.001$. (D and $\mathbf{E}$ ) Coinfected hLEC samples were processed for CLEM to confirm at the ultrastructural level that $M$. tuberculosis $\triangle$ RD1-GFP cords were indeed present in the cytosol (E; magnifications of regions indicated in D, asterisks mark cytosolic bacteria). PDIM, phthiocerol dimycocerosate; hLECs, images of human lymphatic endothelial cells; pi, postinfection; CLEM, correlative light electron microscopy.

little evidence regarding the role of intracellular cords in $M$. tuberculosis pathogenesis. We show here that $M$. tuberculosis intracellular cords are a size-dependent mechanism of evasion of endothelial host cell intracellular innate immune defenses such as xenophagy. We postulate that cords are linked to virulence because bacteria can replicate to a large extent intracellularly within nonimmune cells in a protected environment until nutrients are exhausted and space to grow is limited. Mycobacteria are then released into the extracellular milieu where large cords can block phagocytic uptake, allowing dissemination of M. tuberculosis. This is similar to the extracellular cords that form in the $M$. abscessus-infected zebrafish model, where cords are too large to be phagocytized and therefore facilitate immune evasion (8). We determined that intracellular cording is a result of evading the host cell defenses and allows vast numbers of bacteria to proliferate, only being stopped by physical space and eventually leading to the cell being compromised and cords disseminating, which are too large for phagocytosis by macrophages and/or neutrophils.

High burdens of cytosolic bacteria without induction of host cell death were surprising and suggested that human endothelial cells respond differently to infection in human primary macrophages (29). Several pathological studies have shown that whereas some bacilli produce massive tissue damage, especially in the lungs, others persist in many tissues with no gross evidence of damage (34). We propose that infection in macrophages tends to induce necrotic cell death, whereas endothelial cells are more resistant to cell death and permissive for $M$. tuberculosis growth. Consistent with the prolonged survival of $M$. tuberculosis-infected hLECs, there is an $M$. tuberculosis-induced transcriptional signature of cell death present, but this is alongside the upregulation of several prosurvival pathways. Our studies are consistent with early observations on HeLa cells, in which it was found that only fully virulent $M$. tuberculosis strains could cord, often filling the whole cell without causing cytotoxicity (10). Finally, our data provide an explanation for the observation that endothelial cells are infected in patients with TB, but the typical clinical symptoms of endothelial damage are not observed as in other infectious diseases.

Our study also sheds some light on the preferred site of replication of $M$. tuberculosis in endothelial cells. Our experiments clearly show that if bacteria access the cytosol, then they can cord and replicate. This suggests that the environment in membrane-bound compartments is restrictive and the cytosol is highly permissive for bacterial replication and cording. It remains to be defined if this is the case for macrophages. Interestingly, in one study that investigated the localization of $M$. tuberculosis in resected lungs of patients with TB, prominent cords were observable within macrophages at the luminal side of the granuloma cavity (35). It remains to be determined whether our studies in human cells and tissues would produce similar results in mice; however, the evasion of xenophagy by intracellular cording might provide an explanation for the reported evasion of this pathway in the mouse model of TB (21).

What determines that a subpopulation of intracellular $M$. tuberculosis starts cording? It is possible that differential expression of $M$. tuberculosis-secreted or cell-surface proteins causes differential recognition of cytosolic $M$. tuberculosis by the autophagy apparatus. Modification affecting the synthesis of the bacteria wall component such as trehalose dimycolate is important for cording in vitro and bacterial virulence in vivo $(3,36)$. Here, we observed that individual bacteria forming intracellular cords were smaller than membrane-bound noncording bacteria, suggesting that the localization of those 2 bacterial populations may differ at the transcriptional level. We can speculate that the lower volume of individual bacteria forming intracellular cords is the result of modification in the bacterial wall composition, improving the ability of bacteria to form cords within the cytosol of hLECs and modulate the recognition by cytosolic sensors. Our data show that the previously reported ubiquitin-mediated autophagy process, by which $M$. tuberculosis extracellular DNA/RNA is recognized by the cGAS/STING pathway (17), is also activated in hLECs. Whether it is the bacteria themselves that are ubiquitinated or their compartment is uncertain. If $M$. tuberculosis retains its waxy cell wall in the cytosol, then it is unlikely that ubiquitination will play a major role in xenophagic targeting. We reason that if the bacteria themselves are being recognized, then why is only a subpopulation targeted to autophagy? What is different about them? We hypothesize that it is the ESX-1-mediated damaged membranes surrounding the bacteria that are recognized, and if $M$. tuberculosis is in close proximity to this, then it will be "captured" with it. This process 
A

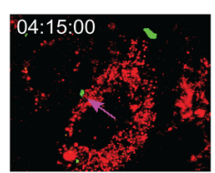

Mtb p62

B

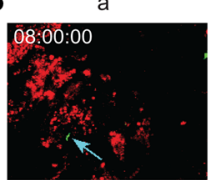

Mtb p62

C

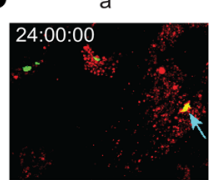

Mtb p62

D

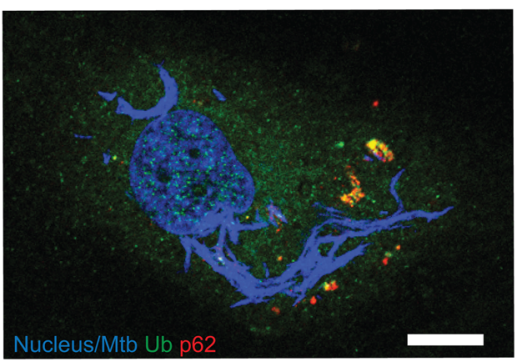

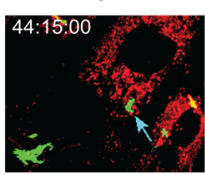

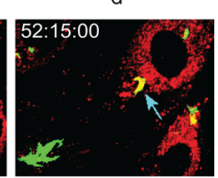

e
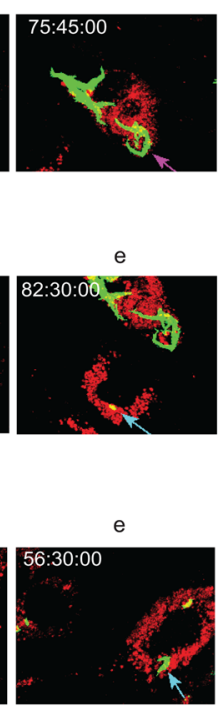
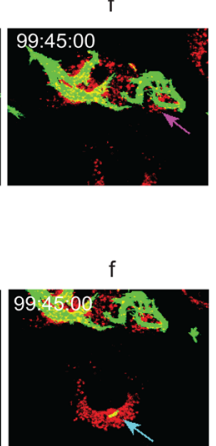
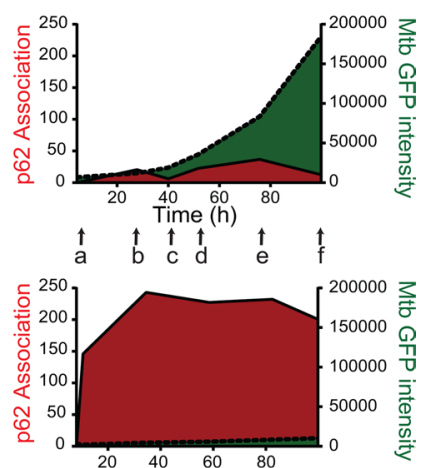

$20 \quad 40 \quad 60 \quad 80$

$\begin{array}{lllll}\text { At } & \uparrow & \uparrow & \uparrow & \uparrow \\ a b & c & d & e & f\end{array}$
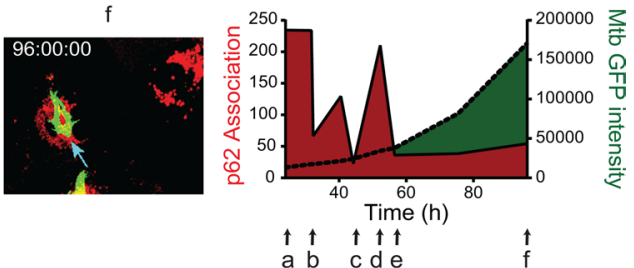

F

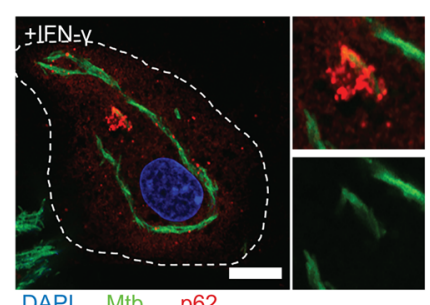

E

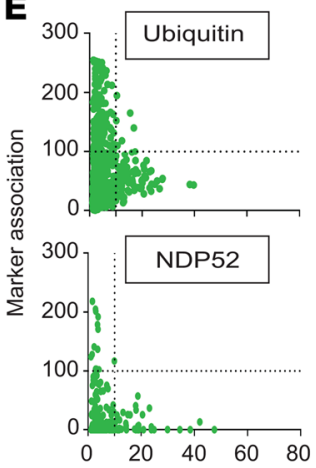

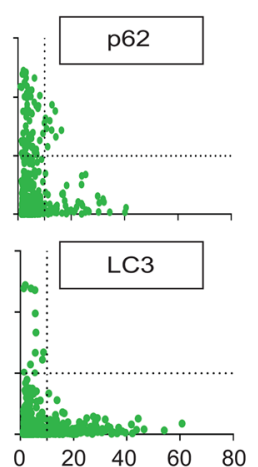

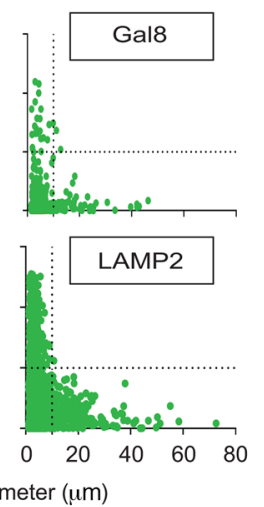

Feret diameter $(\mu \mathrm{m})$ $\begin{array}{lll}t & \uparrow & \uparrow \\ \mathrm{a} & \mathrm{b} & \mathrm{c} d \mathrm{~d}\end{array}$

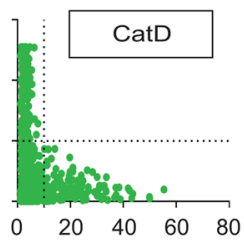

G
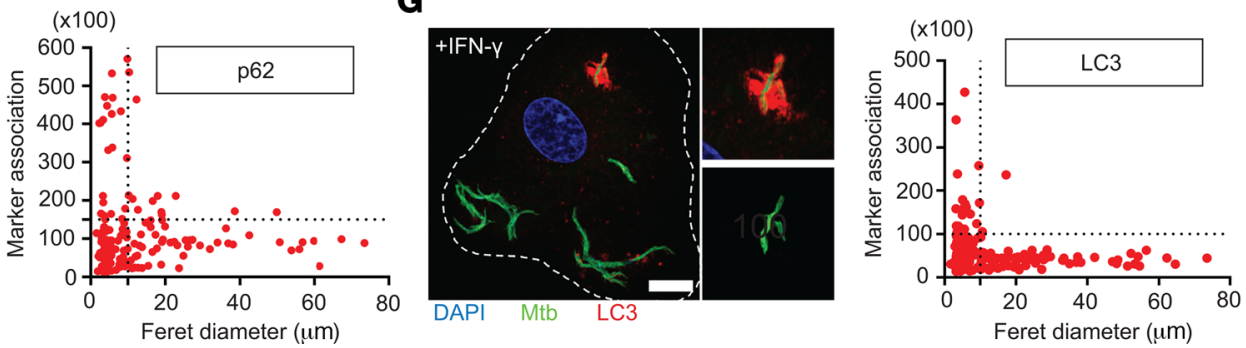

Figure 6. Intracellular cords are not recognized by cytosolic immune sensors in both resting and IFN- $\gamma$-activated hLECs. (A-C) Live-cell imaging of hLECs expressing p62-RFP (red) infected with M. tuberculosis-GFP (green) for 115 hours. Imaging started 15 minutes after addition of the bacteria to the cells. Snapshots from the movies (Supplemental Videos 1-3) are shown, with the time point displayed above in hh:mm:ss format. Scale bars: $10 \mu \mathrm{m}$. (A) The pink arrow tracks an example of an intracellular cord forming from a single bacterium, which never interacts/associates with p62. (B) The blue arrow tracks an example of an individual M. tuberculosis bacterium becoming associated to p62 throughout which leads to restriction of growth. (C) The blue arrow tracks an example of $M$. tuberculosis associating and dissociating with p62 multiple times. Only after p62 association ceased completely, cord formation started. (C-E, right-hand side of panel) NIH Image) software quantification of the GFP intensity and the p62-RFP association of the arrowed bacteria over time. Letters a-f refer to the snapshots in A-C. (D) Representative image of hLECs infected with M. tuberculosis WT-EGFP (blue) for 72 hours and stained for the autophagy adaptor p62 (red) and the autophagy receptor ubiquitin (Ub) (green). Cell nuclei are stained with DAPI (blue). Scale bar: $10 \mu \mathrm{m}$. (E) Intracellular markers of autophagy pathogen sensing were assessed for their association to intracellular cords 72 hours after infection. Particles with a Feret diameter greater than $10 \mu \mathrm{M}$ were considered cords, and a marker association score above 100 was considered positive. Points correspond to individual bacterial particles. (F and G) Representative image of hLECs infected with M. tuberculosis WT-EGFP (green) for 72 hours to let bacteria form intracellular cords before being treated or not with $200 \mathrm{ng} / \mathrm{mL}$ of human IFN- $\gamma$ for an additional 24 hours. Cells were then fixed and stained for the autophagy adaptor p62 (F, left) or LC3 (G, left). Both p62 and LC3 markers are visualized in red; cell nuclei are stained with DAPI (blue). Scale bar: $10 \mu \mathrm{m}$. Intracellular markers p62 (F, right) and LC3 (G, right) were assessed for their association to intracellular bacteria in function of the size of the bacterial cluster. Particles with a Feret diameter greater than $10 \mu \mathrm{M}$ were considered cords, and marker association scores above 15,000 for p62 and 10,000 for LC3 were considered positive. (D-C) Data were obtained from 3 independent experiments, each performed in duplicate. hLECs, images of human lymphatic endothelial cells. 
may be cyclical, with $M$. tuberculosis then damaging the autophagic compartment to escape again. However, if M. tuberculosis can get away from the damaged membranes after cytosolic translocation, then it may be able to evade autophagic capture. This is likely to occur with the majority of the $M$. tuberculosis population and hence why only a relatively small population is targeted to autophagy. It is unlikely that dead bacteria or those that do not damage the phagosomal membrane will be targeted to autophagy because they are ESX-1 and PDIM dependent; these populations are thus likely to mature into phagolysosomes. Although the cording phenotype seems to be unique for pathogenic mycobacteria, it remains to be determined if other cytosolic pathogens also evade autophagy in a size-dependent manner as shown here.

\section{Methods}

\section{Cells}

Primary hLECs taken from inguinal lymph nodes (ScienCell Research Laboratories, catalog 2500) were cultured according to the manufacturer's instructions up to passage 5 as described fully in ref. 19. For confocal microscopy of fixed cells, 20,000 cells in $300 \mu \mathrm{L}$ complete endothelial cell medium (ECM) (ScienCell Research Laboratories, catalog 1001) were seeded onto 10-mm diameter 1.5 glass coverslips (Glaswarenfabrik Karl Hecht, 1001/10_15). For imaging destined for CLEM, 10,000 cells per dish (MatTek, P35G-1.5-14-CGRD) in $500 \mu \mathrm{L}$ ECM were seeded to achieve a confluence of $30 \%-50 \%$ (thus allowing visualization of the grid reference etched into the dish). For live-cell imaging, 25,000 cells per dish in 500 $\mu \mathrm{L}$ ECM were seeded to achieve a confluence of $>80 \%$ (thus limiting the cells' movement away from the field of view). For electron microscopy, 200,000 cells per T25 flask were seeded in $5 \mathrm{~mL}$ ECM. For imaging with the automated confocal microscope Opera Phenix, 5000 cells per well were seeded in 96-well plate (Cell Carrier 96 ultra, PerkinElmer). Type II alveolar epithelial A549 cells (ATCC) were cultured according to the manufacturer's instructions. For confocal microscopy, 50,000 cells in $500 \mu 1$ DMEM (Gibco) supplemented with $10 \%$ (v/v) heat-inactivated FCS were seeded onto $10-\mathrm{mm}$ diameter 1.5 glass coverslips.

\section{M. tuberculosis strains}

This study used the following EGFP-tagged strains as described previously (19, 30, 31): M. tuberculosis H37Rv-EGFP (M. tuberculosis WT) and M. tuberculosis H37Rv-EGFP $\triangle \mathrm{RD} 1$ (M. tuberculosis $\triangle \mathrm{RD} 1)$. In this study, we refer to the $M$. tuberculosis-GFP WT strain as $M$. tuberculosis WT and the $M$. tuberculosis-GFP PMM100 strain as $M$. tuberculosis $\triangle$ PDIM. Additionally, we have used M. tuberculosis H37Rv-RFP (tagged with plasmid pML2570) and H37Rv- $\triangle$ RD1-E2-Crimson (tagged with plasmid pTEC19, which was a gift from Lalita Ramakrishnan (University of Cambridge, Cambridge, United Kingdom); Addgene plasmid 30178) (37). The strains had been routinely tested for PDIM expression as shown in Supplemental Figure 3. The protocol is described in the Supplemental materials. The clinical isolates $M$. tuberculosis N0072-EGFP (lineage 1), $M$. tuberculosis N0145-EGFP (lineage 2), and M. tuberculosis N0024-EGFP (lineage 3) were obtained from Sebastien Gagneux (Swiss TPH, Basel, Switzerland). Mycobacteria were cultured in Middlebrook's 7H9 broth medium (MilliporeSigma, M0178) and supplemented with 10\% (v/v) Middlebrook OADC (BD Biosciences, catalog 212351) and 0.05\% (v/v) Tween80 (MilliporeSigma, P1754) in $50 \mathrm{~mL}$ Falcon tubes at $37^{\circ} \mathrm{C}$ with rotation. Alternatively, mycobacteria were plated on Petri dishes containing Middlebrook's 7H11 agar medium (MilliporeSigma, M0428) and supplemented with $10 \% \mathrm{OADC}$, then incubated at $37^{\circ} \mathrm{C}$ for 2 to 3 weeks until colonies appeared.

\section{Infection of hLECs with M. tuberculosis}

A detailed infection protocol can be found in ref. 19. Briefly, M. tuberculosis cultures were grown to midexponential phase and washed twice with PBS, once with ECM medium, then shaken with glass beads to break up bacterial clumps. M. tuberculosis was then resuspended in ECM medium and centrifuged at a slow speed to pellet any remaining clumps but leaving individual bacteria in suspension. The $\mathrm{OD}_{600}$ of the bacterial suspension was measured and then added to hLECs at a theoretical MOI of 10 in ECM medium. Infection was for 5 hours and followed by 2 PBS washes to remove any uninfected M. tuberculosis. The infected cells were incubated usually for 2 to 72 hours but up to 7 days for live-cell imaging. For experiments requiring coinfection of $2 M$. tuberculosis strains, we used strains tagged with different colors to distinguish between them (RFP, EGFP, or E2-Crimson). These strains were prepared individually using the above-mentioned method and mixed only just before hLEC infection (at an MOI of 5 each, to achieve a total MOI of 10$)$. 
For the activation of hLECs with IFN- $\gamma$ after infection, hLECs were infected with $M$. tuberculosis at an MOI of 5 for 72 hours, as described below. After 72 hours, human IFN- $\gamma$ (PeproTech, catalog 300-02) was added in appropriate wells at a final concentration of $200 \mathrm{ng} / \mathrm{mL}$. Cells were then incubated for an additional 24 hours before being fixed with a PBS-PFA 4\% solution and processed for immunofluorescence.

\section{Indirect immunofluorescence}

An extended method can be found in ref. 19. In summary, infected hLECs on coverslips were fixed with 3\% methanol-free paraformaldehyde (Electron Microscopy Sciences, catalog 15710) in PBS for 24 hours. Coverslips were quenched with $50 \mathrm{mM} \mathrm{NH}_{4} \mathrm{Cl}$ (MilliporeSigma, A9434) and then permeabilized with $0.01 \%$ saponin (MilliporeSigma, 84510) 1\% BSA (MilliporeSigma, A3912) in PBS. The cells were washed with PBS, then 30 to $50 \mu \mathrm{L}$ of the primary antibody (diluted in PBS with $0.01 \%$ saponin, $1 \%$ BSA) was added onto the coverslips for 1 to 2 hours at room temperature (detailed in Supplemental Table 1). Following this, 3 PBS washes preceded the addition of the secondary antibody (diluted in the same way as the primary antibody) for 1 hour at room temperature. The coverslips were again washed 3 times in PBS, before an optional staining step for F-actin using a 1:250 dilution of rhodamine phalloidin (Biotium, catalog 00027), Alexa Fluor 633 phalloidin (Life Technologies, catalog A22284), or Alexa Fluor 488 phalloidin (Life Technologies, catalog A12379) for 20 minutes at room temperature. After 3 more PBS washes, 300 nM DAPI (Life Technologies, catalog D3571) in PBS was added for 10 minutes to stain nuclei. After a final PBS wash, the coverslips were mounted onto glass slides using mounting medium (DAKO Cytomation, S3023).

\section{Confocal microscope image acquisition and analysis}

Imaging of fixed samples was performed using a Leica SP5 AOBS Laser Scanning Confocal Microscope(Leica Microsystems) exactly as detailed in ref. 19. Images were obtained in.lif format and imported into Fiji (NIH). Three parameters were measured using Fiji: (a) M. tuberculosis growth using the total GFP signal per hLEC; (b) association of a marker (e.g., Galectin 8) to M. tuberculosis; and (c) intracellular cord size using Feret diameter. (a) and (b) are described extensively in ref. 19, whereas Feret diameter is explained in Supplemental Figure 1. All data were plotted and analyzed using Microsoft Excel 2010, GraphPad Prism 6, or ggplot2 (Hadley Wickham) in R (The R Project for Statistical Computing).

\section{Automated confocal microscope image acquisition and analysis}

After infection in a 96-well plate, cells were fixed and stained with DAPI and then fluorescently labeled phalloidin (conjugated with Alexa Fluor 633 or Alexa Fluor 488). Images were acquired using an automated fluorescent confocal microscope (OPERA Phenix, PerkinElmer) equipped with a $\times 63$ (NA 1.2) water lens and 405-, 488-, 561-, and 640-nm excitation lasers. The emitted fluorescence was captured using 2 cameras associated with a set of filters covering a detection wavelength ranging from 450 to $690 \mathrm{~nm}$. For each well, 30-35 adjoining fields containing $4 Z$-stacks separated by $1 \mu \mathrm{m}$ were acquired. We applied a $10 \%$ overlap between fields to generate a global image clustering all the fields in a single image. The maximum projection of the images was analyzed using a dedicated in-built script developed using the image analysis software Harmony 4.6 (PerkinElmer).

Cell segmentation. A local intensity detection algorithm applied on the DAPI channel was used to detect both nuclei and cytoplasm (nuclei: maximal local intensity; cytoplasm: minimal local intensity).

Intracellular bacteria detection. A spot detection algorithm based on the GFP, RFP, or Far Red channel (according to the fluorophore expressed by the bacterial strains) was applied for the detection of intracellular fluorescent $M$. tuberculosis H37Rv (WT), H37Rv- $\triangle \mathrm{PDIM}$, or H37Rv- $\mathrm{RD} 1$. A manual threshold method, using noninfected wells, was applied to determine the background threshold. These spots were defined as region of interest for the measurement of bacterial intensity and area in pixels. The relative bacterial load was expressed in bacterial area (pixel) per cell. The intracellular bacterial growth was quantified by the ratio of intracellular bacterial area per cell between T0 ( 5 hours after infection $=$ uptake) and 3 days after infection. For the quantification of the Feret diameter, the global image of the bacteria channel was exported from Harmony in.png before being converted in 8-bit image and analyzed in Fiji as previously described (ref. 19 and Supplemental Figure 1). 


\section{Live-cell imaging}

hLECs were seeded and infected as previously described. Twenty-four hours before infection, the cells were transduced with LentiBrite RFP-p62 Lentiviral Biosensor (Merck Millipore, catalog 17-10404) using an MOI of 40 according to the manufacturer's instructions. After infecting hLECs, the live-cell dishes were placed in a holder custom-made for confocal microscopy in a biosafety level 3 laboratory and imaged using the following conditions: 15 -minute frame intervals, $Z$-stacks of 5 slices with 1.38$\mu \mathrm{m}$ thickness, line averaging 4 , and zoom of 1 .

\section{Electron microscopy of single-infected cells}

Electron microscopy was performed exactly as previously described (19). Briefly, hLECs were infected for $5+72$ hours with $M$. tuberculosis WT-EGFP before fixation in 4\% PFA/2.5\% GA in $0.1 \mathrm{M}$ phosphate buffer for 24 hours at $4^{\circ} \mathrm{C}$. The field of view of interest was imaged first by confocal microscopy and then processed for imaging by SBF scanning electron microscopy (SEM) using a 3View2XP attached to a Sigma VP SEM (Zeiss). The same field of view was captured, thus facilitating the creation of a composite CLEM image. SBF SEM images were collected at $1.8 \mathrm{kV}$ using the high current setting with a $20-\mu \mathrm{m}$ aperture at 5 to $10 \mathrm{~Pa}$ chamber pressure and a $2 \mu \mathrm{s}$ dwell time. Maximum intensity projections of confocal slices were aligned manually to highlight bacteria positions.

\section{Measurement of intracellular M. tuberculosis volumes}

Selected bacteria were segmented manually from slices of SBF SEM data sets and 3D reconstructions were made using the $3 \mathrm{dmod}$ program of IMOD (38). Each data set was first denoised with a 0.5 -pixel Gaussian blur filter applied in Fiji (NIH ImageJ software). Two data sets from each of 2 independent samples were then segmented for each of the cord and membrane-bound bacteria conditions. The data set $x y$ pixels were $9.9 \mathrm{~nm}$ and $8.7 \mathrm{~nm}$ for cord bacteria, and $5.4 \mathrm{~nm}$ and $6.3 \mathrm{~nm}$ for membrane bound bacteria; all data sets consisted of serial images of 50-nm thickness. The data set dimensions were 81.1 $\times 81.1 \times 5.55,71.3 \times 71.36 \times 1,22.1 \times 22.1 \times 1.55$, and $51.6 \times 51.6 \times 2.75 \mu \mathrm{m}$ in $x y z$, with 111,20 , 31 , and 55 serial images, respectively. To calculate bacterial volumes, IMOD calculated the volume inside the mesh using 3D mesh structures derived from closed contours drawn around bacteria each 50 $\mathrm{nm}$, using imodmesh. For CLEM of representative 3D reconstructions of bacteria, an SBF SEM slice was assigned to a confocal slice manually in $z$. The confocal slice was then processed in Fiji: first, to improve interpolation during TurboReg alignment, the confocal image was upscaled from 1024 to 2048 pixels with a bilinear interpolation, and a smooth filter was applied twice; then TurboReg was used to align the processed confocal slice with the SBF SEM image using a scaled rotation transformation and bacteria as landmarks (identified by fluorescence and morphology). The remaining SBF SEM images in the stack were further denoised with a 1-pixel Gaussian blur filter and brightness/contrast adjusted to match the CLEM image in Photoshop. The CLEM image was then inserted into the stack, and a snapshot was taken of the bacterial segmentation with the stack in the model view of $3 \mathrm{dmod}$.

\section{CLEM of coinfected cells}

hLECs were coinfected with $M$. tuberculosis WT-RFP and $M$. tuberculosis $\triangle$ RD1-GFP before fixation and confocal microscopy as above. The field of interest was then processed for imaging by transmission electron microscopy (TEM). The cells were post-fixed in $1 \%$ reduced osmium tetroxide, stained with tannic acid, and quenched in $1 \%$ sodium sulphate. Next, the cells were dehydrated progressively up to $100 \%$ ethanol and incubated in a 1:1 propylene oxide/epon resin mixture. After infiltrations in pure resin, the samples were embedded at $60^{\circ} \mathrm{C}$ for 24 hours. SBF SEM and TEM were performed as described previously (39). Briefly, the field of interest was approached by SBF SEM (there being sufficient signal for approach imaging even though the cells were not processed for this method), then the cut face was aligned to a diamond knife in a UC7 ultramicrotome (Leica Microsystems) and 70- to 80-nm sections from the field of interest were collected. The sections were stained with lead citrate and imaged in a TEM (Tecnai G2 Spirit BioTwin; Thermo Fisher Scientific) using a charge-coupled device camera (Orius; Gatan Inc.). For CLEM overlay, TEM images were assigned to confocal slices manually in $z$. The confocal slice was then processed and aligned with TurboReg in Fiji as above. 
Histology, immunohistochemistry, and analysis

Formalin-fixed paraffin-embedded cervical lymph node tissue sections from patients diagnosed as TB culture positive and/or acid-fast bacilli positive $(\mathrm{AFB}+)$ were selected for the study and processed as described before (19). Briefly, tissue sections were deparaffinized for 10 minutes in xylene and then in $100 \%, 95 \%$, and $80 \%$ ethanol for 2 minutes each. Tissue sections were then placed into an antigen retrieval buffer (Access Super Antigen Solution, Menarini Diagnostics) in a decloaking chamber (Biocare Medical), incubated at 110 degrees for 10 minutes, and allowed to cool for 60 minutes. Sections were permeabilized in PBS $0.2 \%$ Triton X-100 and incubated in blocking buffer (1\% BSA, 5\% FCS in PBS) overnight at room temperature. Tissue sections were labeled using primary antibodies rabbit anti-M. tuberculosis (Menapath, 1:100 dilution) and rat anti-PDPN (BioLegend, catalog 337002, 1:200 dilution). Secondary antibodies used were a goat anti-rabbit Alexa Fluor 488 and a goat anti-rat Alexa Fluor 568 (Life Technologies, catalog A11077 and catalog A11034, respectively, dilution 1/800). Primary and secondary antibodies were tested for cross-reaction in samples of uninfected individuals. Primary (human antigens) and secondary antibodies were tested for cross-reaction with $M$. tuberculosis in samples that were $\mathrm{AFB}^{+}$.

\section{RNA extraction and sequencing library preparation}

M. tuberculosis-infected or uninfected hLECs (48 hours infection) were lysed in $0.5 \mathrm{~mL}$ TRIzol and RNA was extracted using Direct-zol RNA MiniPrep Kit (Zymo Research) and treated with TURBO DNase I (Life Technologies) until DNA free. Quantity and quality of the extracted RNA were determined by Qubit flourometer, NanoDrop spectrophotometer, and Bioanalzyer. RNA-Seq libraries were prepared using $1 \mathrm{mg}$ of RNA of each sample with TruSeq Stranded Total RNA Library Prep kit (Illumina) and ribosomal RNA was removed with Ribo-Zero as part of the library construction process. Quality and quantity of the cDNA libraries were determined by Qubit flourometer and Bioanalzyer before being processed for sequencing with Illumina Hi-Seq 2500 for single-end reads with 100 cycles.

\section{RNA-Seq data analysis}

The RNA-Seq data in this paper have been deposited in Gene Expression Omnibus repository (accession GSE110564). The quality of the Illumina-produced fastq files was assessed using FastQC (v0.11.5) and adapter trimmed using Trimmomatic (v0.36). The resulting reads were then aligned to the human genome (Ensembl GRCh38 release 88 build) using STAR aligner (v2.5.2a). Gene counting was done using RSEM (v1.2.29) and expected read counts were normalized using DESeq2 (v1.18.1), which also determined the $\log _{2}$ fold change and statistical significance between the infected and uninfected samples. Canonical pathway and functional process analyses were performed using IPA (QIAGEN; www.qiagenbioinformatics. com/products/ingenuity-pathway-analysis/) and MetaCore (Thomson Reuters). The expression of several genes had been confirmed by RT-qPCR as described in Supplemental materials.

\section{Statistics}

Results are expressed as mean \pm SEM. All statistical analyses were performed in Prism 6 (GraphPad Software). Means between 2 groups were compared using 2-tailed Student's $t$ tests and means among 3 or more groups were compared using 1-way ANOVA with Tukey's multiple comparisons tests. A $P$ value of less than 0.05 was considered significant. Plots were produced in Prism 6 or ggplot 2 in $\mathrm{R}$ (The R Project for Statistical Computing).

\section{Study approval}

The study was performed using excised cervical lymph node tissue stored within the Department of Anatomical Pathology at Groote Schuur Hospital (Cape Town, South Africa). All of these biopsies were taken for clinical indications. Residual paraffin-embedded blocks of these specimens were stored for further processing. This study complied with the Declaration of Helsinki (2008), and ethics approval was obtained from the University of Cape Town Human Research Ethics Committee (REC187/2013). Informed consent was waived, as this was a retrospective study of formalin-fixed paraffin-embedded tissue samples collected during the course of routine clinical practice. Patient identifiers were unavailable to investigators. 


\section{Author contributions}

MGG and TRL conceived the project. MGG, TRL, CJQ, and RPL designed the experiments. TRL, CJQ, RPL, MRGR, and AF performed experiments. TRL, CJQ, RPL, MRGR, AF, LC, DJG, and RJW analyzed data and provided intellectual input. MGG wrote the manuscript with input from TRL and CJQ. All authors read the manuscript and provided critical feedback.

\section{Acknowledgments}

We thank Michael Niederweis (University of Alabama) for fluorescent plasmids. We thank Bill Jacobs (Albert Einstein College of Medicine), Suzie Hingley-Wilson (University of Surrey), Catherine Astaire-Dequeuer (Institute Of Pharmacology Structural Biology, Toulouse), Douglas Young (Francis Crick Institute, London), and Sebastien Gagneux (Swiss TPH) for M. tuberculosis strains; Steve Coade for assistance with fluorescent tagging of clinical isolates; and Susanne Herbst for critical reading of the manuscript. This work was supported by the Francis Crick Institute (to MGG and RJW), which receives its core funding from Cancer Research UK (FC001092, FC00110218), the UK Medical Research Council (FC001092, FC00110218), and the Wellcome Trust (FC001092, FC00110218) and Wellcome Trust (to RJW, 104803, 203135).

Address correspondence to: Maximiliano G. Crick, Host-pathogen interactions in tuberculosis laboratory, The Francis Crick Institute, 1 Midland Road, London, NW1 1AT, United Kingdom. Phone: 44.0.203 7961460; Email: max.g@crick.ac.uk.

1. [No authors listed]. Global tuberculosis report 2019. World Health Organization. https://www.who.int/tb/publications/ global_report/en/. Published October 19, 2019. Accessed April 21, 2020.

2. Koch R. Die Äetiologie der Tuberkulose. Berliner klinische Wochenschrift. 1882;15: 221-230.

3. Glickman MS, Cox JS, Jacobs WR. A novel mycolic acid cyclopropane synthetase is required for cording, persistence, and virulence of Mycobacterium tuberculosis. Mol Cell. 2000;5(4):717-727.

4. Middlebrook G, Dubos RJ, Pierce C. Virulence and morphological characteristics of mammalian Tubercle bacilli. J Exp Med. $1947 ; 86(2): 175-184$.

5. Hunter RL, Olsen MR, Jagannath C, Actor JK. Multiple roles of cord factor in the pathogenesis of primary, secondary, and cavitary tuberculosis, including a revised description of the pathology of secondary disease. Ann Clin Lab Sci. 2006;36(4):371-386.

6. Hunter RL, Venkataprasad N, Olsen MR. The role of trehalose dimycolate (cord factor) on morphology of virulent M. tuberculosis in vitro. Tuberculosis (Edinb). 2006;86(5):349-356.

7. Indrigo J, Hunter RL, Actor JK. Influence of trehalose 6,6'-dimycolate (TDM) during mycobacterial infection of bone marrow macrophages. Microbiology (Reading, Engl). 2002;148(pt 7):1991-1998.

8. Bernut A, et al. Mycobacterium abscessus cording prevents phagocytosis and promotes abscess formation. Proc Natl Acad Sci U S A. 2014;111(10):E943-E952.

9. Maximow A. Étude comparative des cultures de tissus inoculées soit avec le bacille tuberculeux du type bovin soit avec le bacille BCG de Calmette-Guerin. Annis Inst Pasteur. 1928;42:225-245.

10. SHEPARD CC. Growth characteristics of tubercle bacilli and certain other mycobacteria in HeLa cells. J Exp Med. 1957;105(1):39-48.

11. Ferrer NL, et al. Intracellular replication of attenuated Mycobacterium tuberculosis phoP mutant in the absence of host cell cytotoxicity. Microbes Infect. 2009;11(1):115-122.

12. Kalsum S, et al. The cording phenotype of Mycobacterium tuberculosis induces the formation of extracellular traps in human macrophages. Front Cell Infect Microbiol. 2017;7:278.

13. Wong KW, Jacobs WR. Mycobacterium tuberculosis exploits human interferon $\gamma$ to stimulate macrophage extracellular trap formation and necrosis. J Infect Dis. 2013;208(1):109-119.

14. Galluzzi L, et al. Molecular definitions of autophagy and related processes. EMBO J. 2017;36(13):1811-1836.

15. Deretic V, Levine B. Autophagy, immunity, and microbial adaptations. Cell Host Microbe. 2009;5(6):527-549.

16. Gutierrez MG, Master SS, Singh SB, Taylor GA, Colombo MI, Deretic V. Autophagy is a defense mechanism inhibiting BCG and Mycobacterium tuberculosis survival in infected macrophages. Cell. 2004;119(6):753-766.

17. Watson RO, Manzanillo PS, Cox JS. Extracellular M. tuberculosis DNA targets bacteria for autophagy by activating the host DNA-sensing pathway. Cell. 2012;150(4): 803-815.

18. Deretic V, et al. Autophagy in immunity against Mycobacterium tuberculosis: a model system to dissect immunological roles of autophagy. Curr Top Microbiol Immunol. 2009;335:169-188.

19. Lerner TR, et al. Lymphatic endothelial cells are a replicative niche for Mycobacterium tuberculosis. J Clin Invest. 2016;126(3):1093-1108.

20. Romagnoli A, et al. ESX-1 dependent impairment of autophagic flux by Mycobacterium tuberculosis in human dendritic cells. Autophagy. 2012;8(9):1357-1370.

21. Kimmey JM, et al. Unique role for ATG5 in neutrophil-mediated immunopathology during M. tuberculosis infection. Nature. 2015;528(7583):565-569.

22. Behr MA, Waters WR. Is tuberculosis a lymphatic disease with a pulmonary portal? Lancet Infect Dis. 2014;14(3):250-255.

23. Ganchua SKC, et al. Lymph nodes are sites of prolonged bacterial persistence during Mycobacterium tuberculosis infection in 
macaques. PLoS Pathog. 2018;14(11):e1007337.

24. Patterson KC, Queval CJ, Gutierrez MG. Granulomatous inflammation in Tuberculosis and Sarcoidosis: Does the lymphatic system contribute to disease? Bioessays. 2019;41(11):e1900086

25. Ganbat D, et al. Mycobacteria infect different cell types in the human lung and cause species dependent cellular changes in infected cells. BMC Pulm Med. 2016;16:(19).

26. Lerner TR, Borel S, Gutierrez MG. The innate immune response in human tuberculosis. Cell Microbiol. 2015;17(9):1277-1285

27. Randall PJ, Hsu NJ, Quesniaux V, Ryffel B, Jacobs M. Mycobacterium tuberculosis infection of the 'non-classical immune cell'. Immunol Cell Biol. 2015;93(9):789-795.

28. Nair VR, et al. Microfold cells actively translocate Mycobacterium tuberculosis to initiate infection. Cell Rep. 2016;16(5):1253-1258

29. Lerner TR, et al. Mycobacterium tuberculosis replicates within necrotic human macrophages. J Cell Biol. 2017;216(3):583-594.

30. Lerner TR, Queval CJ, Fearns A, Repnik U, Griffiths G, Gutierrez MG. Phthiocerol dimycocerosates promote access to the cytosol and intracellular burden of Mycobacterium tuberculosis in lymphatic endothelial cells. BMC Biol. 2018;16(1):1.

31. Astarie-Dequeker C, et al. Phthiocerol dimycocerosates of M. tuberculosis participate in macrophage invasion by inducing changes in the organization of plasma membrane lipids. PLoS Pathog. 2009;5(2):e1000289.

32. Augenstreich J, et al. ESX-1 and phthiocerol dimycocerosates of Mycobacterium tuberculosis act in concert to cause phagosomal rupture and host cell apoptosis. Cell Microbiol. 2017;19:(7).

33. Quigley J, Hughitt VK, Velikovsky CA, Mariuzza RA, El-Sayed NM, Briken V. The cell wall lipid PDIM contributes to phagosomal escape and host cell exit of Mycobacterium tuberculosis. mBio. 2017;8(2):e00148-17.

34. Hunter RL, Hwang SA, Jagannath C, Actor JK. Cord factor as an invisibility cloak? A hypothesis for asymptomatic TB persistence. Tuberculosis (Edinb). 2016;101S:S2-S8.

35. Kaplan G, et al. Mycobacterium tuberculosis growth at the cavity surface: a microenvironment with failed immunity. Infect Immun. 2003;71(12):7099-7108

36. Rao V, Fujiwara N, Porcelli SA, Glickman MS. Mycobacterium tuberculosis controls host innate immune activation through cyclopropane modification of a glycolipid effector molecule. J Exp Med. 2005;201(4):535-543.

37. Takaki K, Davis JM, Winglee K, Ramakrishnan L. Evaluation of the pathogenesis and treatment of Mycobacterium marinum infection in zebrafish. Nat Protoc. 2013;8(6):1114-1124.

38. Kremer JR, Mastronarde DN, McIntosh JR. Computer visualization of three-dimensional image data using IMOD. J Struct Biol. 1996;116(1):71-76.

39. Russell MR, et al. 3D correlative light and electron microscopy of cultured cells using serial blockface scanning electron microscopy. J Cell Sci. 2017;130(1):278-291. 